\title{
Jurys, participation civique et représentation au Québec et au Bas-Canada : les grands jurys du district de Montréal (1764-1832)
}

\section{Donald Fyson}

Volume 55, numéro 1, été 2001

URI : https://id.erudit.org/iderudit/005424ar

DOI : https://doi.org/10.7202/005424ar

Aller au sommaire du numéro

\section{Éditeur(s)}

Institut d'histoire de l'Amérique française

ISSN

0035-2357 (imprimé)

1492-1383 (numérique)

Découvrir la revue

Citer cet article

Fyson, D. (2001). Jurys, participation civique et représentation au Québec et au Bas-Canada : les grands jurys du district de Montréal (1764-1832). Revue d'histoire de l'Amérique française, 55(1), 85-120.

https://doi.org/10.7202/005424ar

\section{Résumé de l'article}

Cette étude se penche sur la transplantation de l'institution des grands jurys au Québec/Bas-Canada. Dans la colonie, la sélection et la représentativité des grands jurys sont biaisées, mais pas selon le découpage classique entre Britanniques/Tories et Canadiens/Patriotes ; c'est plutôt le rang social, la profession et le lieu de résidence qui sont déterminants et les grands jurés représentent avant tout la bourgeoisie commerciale urbaine, canadienne et britannique. Le grand jury est certainement une partie intégrante de l'identité britannique et tory, mais aussi de la notion de citoyenneté des élites réformatrices, qui ne rejettent pas cette institution britannique. Enfin, les grands jurys jouent un rôle politique très important, quoique surtout au niveau local : en l'absence d'institutions municipales représentatives, ils permettent aux élites locales d'influencer une administration agissant directement sur leurs intérêts. 


\section{Jurys, participation civique et représentation au Québec et au Bas-Canada : les grands jurys du district de Montréal (I764-I832) ${ }^{\text {I }}$}

DONALD FYSON

Département d'histoire

Université Laval

RÉSUMÉ - Cette étude se penche sur la transplantation de l'institution des grands jurys au Québec/Bas-Canada. Dans la colonie, la sélection et la représentativité des grands jurys sont biaisées, mais pas selon le découpage classique entre Britanniques/Tories et Canadiens/Patriotes; c'est plutôt le rang social, la profession et le lieu de résidence qui sont déterminants et les grands jurés représentent avant tout la bourgeoisie commerciale urbaine, canadienne et britannique. Le grand jury est certainement une partie intégrante de l'identité britannique et tory, mais aussi de la notion de citoyenneté des élites réformatrices, qui ne rejettent pas cette institution britannique. Enfin, les grands jurys jouent un rôle politique très important, quoique surtout au niveau local : en l'absence d'institutions municipales représentatives, ils permettent aux élites locales d'influencer une administration agissant directement sur leurs intérêts.

ABSTRACT - This study examines the transplantation of the institution of the grand jury to Quebec and Lower Canada. In the colony, the selection and the representativity of the grand juries were biased, but not along the classic fracture of British/Tory versus Canadien/Patriote; rather, social place, profession and residence were determinant, with the grand juries representing above all the urban commercial bourgeoisie, both Canadien and British. The grand jury was certainly an integral part of British and Tory identity, but also of the conception of citizenship of the reform elites, who did not reject this British institution. Finally, grand juries played an important political role, but above all at the local level; in the absence of representative municipal institutions, they allowed local elites to influence an administration that directly affected their interests.

1. Mes remerciements à Jean-Philippe Garneau, Steven Watt et Richard LaRue pour avoir commenté des versions préliminaires, à Jean-Marie Fecteau pour avoir partagé ses sources, aux évaluateurs anonymes pour leurs commentaires et au Conseil de recherches en sciences humaines du Canada pour avoir financé une partie de cette recherche. 
T a place centrale du jury dans l'histoire juridique des pays du com$\triangle$ mon law est incontestable. Avec les juges et les officiers de justice, les jurys sont une des trois composantes fondamentales de la justice étatique anglaise et cela, jusqu'au XIx ${ }^{\mathrm{e}}$ siècle au moins. Du côté civil, les jurys tranchent les litiges entre les partis et fixent les dommages; du côté criminel, les grands jurys évaluent les preuves à charge avant le procès, tandis que les petits jurys déterminent la culpabilité. Dans l'exercice de ces fonctions juridiques formelles, le cercle des jurys devient un lieu privilégié pour l'exercice du pouvoir social et politique, donnant une sanction à saveur populaire à la répression juridique de la dissidence politique et de la déviance sociale ${ }^{2}$.

L'importance sociale et politique du jury déborde largement ses fonctions strictement juridiques, car l'institution est aussi une composante clé des concepts de citoyenneté et des systèmes de gouvernance et de participation civique. D’abord, le jury est un symbole puissant de la citoyenneté britannique. Présenté comme une institution populaire qui garantit les libertés individuelles contre la tyrannie, il occupe une place de choix dans la rhétorique et la théorie constitutionnelles anglaises et demeure fondamental à la construction de l'identité et du civisme britanniques ${ }^{3}$. En partie en raison de la puissance symbolique des jurys, leur sélection reflète aussi les relations de pouvoir plus larges dans la société, notamment la tension constante entre la participation élargie et la domination oligarchique ${ }^{4}$. Toutefois, la fonction civique du jury possède aussi un aspect très concret, car les jurys élargissent la participation à la gouvernance offerte par les structures politiques régulières. Par exemple, depuis l'époque médiévale,

2. Voir par exemple J. S. Cockburn et Thomas A. Green, dir., Twelve Good Men and True: The Criminal Trial Jury in England, 1200-1800 (Princeton, Princeton University Press, 1988); Thomas A. Green, Verdict According to Conscience: Perspectives on the English Criminal Trial Jury, 1200-1800 (Chicago, University of Chicago Press, 1985); Richard D. Younger, The People's Panel: The Grand Jury in the United States, 1634-1941 (Providence, Brown University Press, 1963).

3. La discussion classique du jury appartient à William Blackstone, Commentaries on the Laws of England. Sur le jury et l'identité britannique tels que transplantés aux colonies nord-américaines, voir par exemple Paul Romney, «From Constitutionalism to Legalism: Trial by Jury, Responsible Government, and the Rule of Law in the Canadian Political Culture ", Law and History Review, 7,1(1989) : 121-174; Daniel J. Hulsebosch, «Imperia in Imperio: The Multiple Constitutions of Empire in New York, 1750-1777 ", Law and History Review, 16,2 (1998) : 319-380.

4. Voir en particulier les articles dans J. S. Cockburn et T. A. Green, dir., Twelve Good Men and True..., op. cit.; sur l'Angleterre du xvıII ${ }^{\mathrm{e}}$ siècle, comparez P. J. R. King, "Illiterate Plebeians, Easily Misled”: Jury Composition, Experience and Behavior in Essex, 1735-1815 », 254-304; Douglas Hay, "The Class Composition of the Palladium of Liberty: Trial Jurors in the Eighteenth Century", 305-357. 
des jurys composés de résidants locaux assistent le coroner. Encore plus fondamentalement, une des tâches principales des grands jurys est de présenter leurs constatations sur l'état de leur communauté, pour guider l'intervention judiciaire, administrative ou législative. En somme, dans un système politique et étatique qui concentre la plus grande partie du pouvoir entre les mains d'une élite sociale et politique restreinte, les jurys viennent donner, en théorie, une voix à la communauté locale 5 .

Deux exemples, tirés de l'histoire du jury au Québec, démontrent l'importance du jury au-delà du procès criminel ou civil. En octobre 1764, quelques semaines à peine après l'inauguration de l'administration civile britannique, le grand jury des Sessions trimestrielles de la paix de Québec fait sa première représentation (presentment). D’abord, la représentation dresse une liste de doléances contre l'administration coloniale : l'incompétence de l'établissement judiciaire, les inconvénients de la présence militaire, etc. Au-delà de ces critiques, somme toute assez courantes dans les colonies britanniques, les grands jurés abordent d'autres questions politiques plus locales. Notamment, ils affirment que le grand jury étant la seule institution représentative dans la colonie, ses membres devraient être consultés sur toute loi et recevoir la reddition des comptes publics. En d'autres termes, le grand jury se propose de prendre la place de l'Assemblée promise, mais restée lettre morte. En outre, la représentation affirme que les catholiques ne devraient pas être membres des jurys : la présence des catholiques est répugnante aux droits et libertés sacrés de l'Angleterre parce qu'ils reconnaissent la suprématie du pape. La partie principale de la représentation est signée par quatorze jurés britanniques, tous proches de la communauté marchande (et, semble-t-il, spécialement choisis à cet égard) et sept jurés canadiens. Toutefois, la partie touchant les catholiques n'est signée que par les jurés britanniques.

La représentation soulève un tollé. La communauté canadienne est furieuse. D’une part, la prise de position anticatholique de la représentation tendait à confirmer le statut inférieur des Canadiens. D'autre part, les sept jurés canadiens affirment avoir été manipulés pour les amener à signer une représentation, en anglais, qu'ils ne comprennent pas et dont plusieurs points ne reflètent pas les discussions du grand jury. Les jurés canadiens offrent donc une réfutation de la représentation, point par point;

5. Sur le rôle des jurys dans la gouvernance locale, voir par exemple l'étude classique de Sidney et Beatrice Webb, English Local Government, en particulier I : The Parish and the County et II : The Manor and the Borough (London, Longmans, 1906, 1908). 
toutefois, par rapport au statut représentatif du grand jury, ils se contentent de passer à côté des questions «qui nous paraissent encore fort éloignées par l'espérance ou nous sommes, qu'il ne sera question d'aucune [sic] Impôt dans cette Colonie». Comme les jurés canadiens, les magistrats répondent aussi de manière cinglante : «Nous ne pouvons $[\ldots]$ concevoir comment un petit nombre d'habitants, dont quelques uns ne font qu'arriver, puissent avoir assez d'influence, d'intérêt ou de connoissance dans la Province, pour se regarder eux mêmes, ou pour être regardés par d'autres, comme les représentans d'icelle.» Même le gouverneur Murray dénonce la représentation comme une folie. Enfin, les jurés britanniques préparent à leur tour une réplique, qui maintient leur prétention d'être consultés par la législature, mais qui (en contradiction directe avec la représentation) déclare que leur intention n'était pas d'exclure les catholiques de tous les jurys, mais seulement dans les causes entre protestants ${ }^{6}$.

Le deuxième exemple date de la fin des années 1820 : un rapport d'un comité de l'Assemblée législative du Bas-Canada formé à la fin de 1828 pour examiner diverses pétitions contre l'administration du gouverneur Dalhousie. L'une des plaintes formulées concerne le shérif du district de Montréal, accusé de constituer les jurys criminels, y compris les grands jurys, exclusivement avec des gens de la ville, plutôt qu'avec ceux de tout le district. Le comité, sous la présidence de Denis-Benjamin Viger, dépose son rapport en février 1829. Le rapport conclut que la pratique courante dans les trois districts judiciaires principaux (Montréal, Québec et TroisRivières) est «évidemment contraire aux lois et aux principes d'impartialité» et que «sommer exclusivement les habitans d'un lieu particulier, d'une classe distincte, ne saurait être compatible avec la liberté plus qu'avec la justice impartiale. Il répugne à tous les principes de notre gouvernement et ne pourrait tendre qu'au renversement de la constitution du pays ${ }^{7} . »$

6. Pour le contexte de la représentation, voir A. L. Burt, The Old Province of Quebec (Toronto, McClelland and Stewart, 1968), I : 99-100; André Morel, «La réception du droit criminel anglais au Québec (1760-1892)", Revue Juridique Thémis, 13,2-3 (1976) : 459-460 et 483-484. La représentation elle-même, la réponse des magistrats et la réplique des jurés britanniques ont été publiées dans deux brochures anonymes, les numéros 41562 (en anglais) et 50683 (en français) de la collection de l'Institut canadien de microreproductions historiques. La représentation et la réplique des jurés canadiens sont aussi reproduites dans Adam Shortt et Arthur G. Doughty, Documents Relating to the Constitutional History of Canada, 1759-1791 (Ottawa, Historical Documents Publication Board, 1918), 212-219. Pour la réaction de Murray, voir Archives nationales du Canada (ci-après ANC), MG23 GII1, 17 novembre 1764.

7. Journaux de la Chambre d'Assemblée du Bas-Canada (ci-après JCABC) 38 (1829), Appendice EE. 
Le débat s'insère dans le contexte de l'affrontement plus large entre les Patriotes et le régime autoritaire de Dalhousie. Le contrôle détenu par l'exécutif sur la sélection des jurés devient un autre symbole du despotisme et de la tentative d'exclure les Canadiens de leurs droits démocratiques. Une série de procès hautement politisés pour diffamation et sédition, dans lesquels les grands jurés sont accusés d'être des partisans de l'administration, ne fait rien pour calmer les esprits. Même avant les travaux du comité, l'administration plus conciliante de James Kempt tente de désamorcer le conflit, en ordonnant, dès l'été de 1828, que les jurys soient sélectionnés dans l'ensemble de chaque district. Néanmoins, la sélection des jurés reste à l'ordre du jour, car la directive n'est pas respectée intégralement avant 1830, en particulier dans le district de Montréal. La législature persiste aussi et, en 1832, elle adopte enfin une loi régissant plus strictement la qualification et la sélection des jurés ${ }^{8}$. Cette nouvelle loi précise entre autres la méthode stricte que doivent suivre les shérifs pour que les jurys soient constitués impartialement à partir de l'ensemble des personnes qualifiées.

Ces deux exemples soulèvent des questions fondamentales quant à la place des jurys dans la vie publique. La composition des jurys favorise-t-elle certains groupes? Quels sont les différents concepts de citoyenneté qui sont attachés aux jurys? Bien au-delà de son rôle juridique, le grand jury peut-il fonctionner comme une institution représentative, dotée de fonctions civiques? La situation particulière du Québec suggère enfin une autre piste d'analyse, car le jury est une institution britannique transplantée dans une société majoritairement francophone. Certains ont présenté cette greffe comme ayant plutôt mal réussi sur le plan juridique ${ }^{9}$, mais est-ce bien le cas pour la fonction civique du jury pris dans une acception plus large?

Pour aborder ces différentes questions, je me concentre dans cet article sur un type de jury en particulier : le grand jury. Institution composée surtout de notables locaux, le grand jury incarne en Angleterre la cooptation des élites qui caractérise l'administration locale jusqu'au $\mathrm{XIX}^{\mathrm{e}} \operatorname{siècle}^{10}$.

8. JCABC 39 (1830), Appendice SS ; 2 William IV c.22 (1832).

9. Douglas Hay, "The Meanings of the Criminal Law in Quebec, 1764-1774», dans Louis A. Knafla, dir., Crime and Criminal Justice in Europe and Canada: Essays (Waterloo, Wilfrid Laurier University Press, 1985), 77-110; André Morel, "La réception du droit criminel anglais », 521-523.

10. Sur les grands jurys en Angleterre à l'époque moderne, voir entre autres $\mathrm{B}$. Webb, The Parish and the County, op. cit., 446-456; J. S. Morrill, The Cheshire Grand Jury, 1625-1659: A Social and Administrative Study (Leicester, Leicester University Press, 1976); J. M. Beattie, Crime and the Courts in England, 1660-1800 (Princeton, Princeton University Press, 1986), 318-333; Georges 
Comme dans la plupart des colonies britanniques ${ }^{11}$, l'institution du grand jury est transplantée telle quelle au Québec comme partie intégrante du droit public anglais. Jusqu'aux réformes du début des années 1830, il n’y a que deux modifications législatives concernant les grands jurys. Le Québec et le Bas-Canada, de 1764 jusqu'au début des années 1830, est donc un milieu idéal pour étudier l'implantation de cette institution britannique dans un contexte qui lui était étranger.

Je me pencherai d'abord sur la sélection et la composition des grands jurys, pour juger de leur représentativité. J’aborderai ensuite les attitudes des élites envers le grand jury. Enfin, j'évaluerai leur rôle dans la vie politique et civique au-delà de leurs décisions juridiques. Je laisse donc de côté leur rôle dans le processus judiciaire lui-même; d'autres chercheurs ont déjà commencé à aborder ce sujet qui, indissociable d'une étude de la justice criminelle en général, constitue en soi une étude distincte ${ }^{12}$. Mon travail se fonde surtout sur une analyse attentive des grands jurys dans le district judiciaire de Montréal, mais avec un regard plus large sur l'ensemble de la colonie.

\section{I - LA SÉLECTION ET LA COMPOSITION DES GRANDS JURYS AU QUÉBEC/BAS-CANADA}

La sélection et la composition des jurys en général ont beaucoup attiré l'attention des chercheurs, en particulier ceux qui travaillent sur l'Angleterre. La question est fondamentale : les jurys sont-ils des institu-

Lamoine, dir., Charges to the Grand Jury, 1689-1803 (London, Royal Historical Society, 1992), 3-22 ; P. J. R. King, "IIlliterate Plebeians, Easily Misled”", op. cit.; D. Hay, «The Class Composition of the Palladium of Liberty", op. cit.

11. Sur la transposition du grand jury dans différents contextes coloniaux, voir par exemple Neal Graham, The Courts, Crime and the Criminal Law in Ireland, 1692-1760 (Dublin, Irish Academic Press, 1996); Gwenda Morgan, "Law and Social Change in Colonial Virginia: The Role of the Grand Jury in Richmond County, 1692-1776», Virginia Magazine of History and Biography, 95,4 (1987) : 453-480; Christopher Waldrep, «Egalitarianism in the Oligarchy: The Grand Jury and Criminal Justice in Livingston County, 1799-1808 ", Filson Club History Quarterly, 55,3 (1981): 253-267; Elise Histed, «The Introduction and Use of the Grand Jury in Victoria », Journal of Legal History, 8,2 (1987) : 167-177; Y. K. Lee, «The Grand Jury in Early Singapore (1819-1873)», Journal of the Malaysian Branch of the Royal Asiatic Society, 46,2 (1973) : 55-150. Sur les grands jurys au Canada, voir Nancy Kay Parker, «Reaching a Verdict: The Changing Structure of DecisionMaking in the Canadian Criminal Courts, 1867-1905 ", thèse de doctorat, York University, 1999), 236-288, qui offre aussi un survol des grands jurys dans le Haut-Canada et la Nouvelle-Écosse avant la Confédération.

12. Par exemple, le rôle des grands jurys lors des procès politiques est abordé dans plusieurs articles dans F. Murray Greenwood et Barry Wright, dir., Canadian State Trials: Law, Politics, and Security Measures, 1608-1837 (Toronto, University of Toronto Press, 1997). 
tions dotées d'une large participation populaire ou sont-ils plutôt l'apanage exclusif d'une élite oligarchique? Les débats tournent autour de plusieurs questions reliées, comme le processus de sélection (les critères d'éligibilité, l'identification des individus éligibles et la sélection opérée parmi ces individus), la composition sociale des jurys et la participation répétée de la part des mêmes jurés ${ }^{13}$. Dans le contexte du Québec et du BasCanada, une étude de la composition des grands jurys permet de voir comment une institution britannique est adaptée au contexte colonial, mais elle soulève aussi la question fondamentale de la participation canadienne aux institutions civiques de la colonie.

En Angleterre, la sélection des jurés est une affaire relativement décentralisée et en partie publique. La participation aux jurys est fondée avant tout sur la propriété et le sexe. Avec certaines exceptions, elle est limitée aux propriétaires fonciers sans obligations (freeholders) dont les terrains ont une valeur annuelle d'au moins $£ 10$ ou à certains locataires à long terme (life lease). Les femmes sont exclues, sauf dans le cas particulier du jury des matrones chargées de vérifier une grossesse. Des listes de propriétaires masculins éligibles sont confectionnées par les connétables de chaque hundred (une subdivision administrative), puis affichées sur les portes des églises paroissiales et éventuellement transmises au shérif du comté, qui convoque les jurés. La procédure est loin d'être idéale et souvent teintée de corruption, de favoritisme ou d'inefficacité, mais elle est certainement très décentralisée ${ }^{14}$.

Au Québec et au Bas-Canada, le cadre général ne change pas. Pour chaque séance de chacune des deux cours criminelles principales (deux fois par année pour le Banc du Roi, quatre fois pour les Sessions trimestrielles de la paix), le shérif de chaque district convoque un certain nombre de jurés. À partir des jurés qui se présentent, il constitue le grand et les petits jurys. En revanche, le processus de sélection dans la colonie est très différent.

Même si, dans un premier temps, les critères anglais semblent avoir été intégralement repris dans la colonie, ils furent ensuite modifiés de façon significative. D’abord, comme la représentation de 1764 en fait foi, presque à partir du début du gouvernement civil anglais, les catholiques sont au-

13. Plusieurs des articles dans J. S. Cockburn et T. A. Green, Twelve Good Men and True, op. cit., abordent cette question.

14. Richard Burn, The Justice of the Peace, and Parish Officer (London, 1788), $16^{\mathrm{e}}$ edition, II : 693724 ; P. J. R. King, "Illiterate Plebeians, Easily Misled” ", 258-261 ; D. Hay, "The Class Composition of the Palladium of Liberty", op. cit., 311-329; James C. Oldham, "On Pleading the Belly: A History of the Jury of Matrons », Criminal Justice History, 6 (1985) : 1-64. 
torisés à être membres des jurys, à la différence de l'Angleterre des Test Acts $^{15}$; de fait, aucune discrimination religieuse n'est pratiquée, car dans l'ensemble de notre période, au moins cinq individus juifs sont membres de grands jurys ${ }^{16}$. De plus, en 1787, une ordonnance élargit l'éligibilité aux locataires dont le loyer annuel dépasse $£ 15$, invoquant la justification qu'il est trop difficile de trouver des freeholders dans les villes ${ }^{17}$. Cette mesure, apparemment une simple question d'efficacité administrative, vient en réalité rompre l'ancien lien fondamental entre participation aux institutions publiques et propriété; en ce sens, il préfigure certaines dispositions de l'Acte constitutionnel de 1791 . Une somme de $£ 15$ par année constitue un loyer modeste pour un marchand ou un professionnel; la mesure ouvre donc les jurys à presque tous les membres des élites, que ceux-ci possèdent des propriétés dans la colonie ou non. Par contre, à la différence du droit de vote, la participation aux jurys n'est jamais ouverte aux femmes dans la colonie; cet aspect de la participation civique reste une exclusivité masculine.

Les critères de base ont aussi une signification différente dans le contexte colonial. En Angleterre, le cens d'éligibilité fixé à $£ 10$ exclut plus de $90 \%$ des chefs de ménage. Au Québec et au Bas-Canada, la situation est moins nette. D’une part, les critères de $£ 10$ de propriété ou $£ 15$ de loyer sont certainement plus restrictifs que les critères pour être électeur à partir de 1791. Fixé à $£ 2$ de propriété dans les districts ruraux et $£ 5$ de propriété ou $£ 10$ de loyer dans les villes, le cens électoral fait appel à un électorat constitué de la très grande majorité des propriétaires ruraux et de la majorité des chefs de ménage urbains. La signification des critères d'éligibilité pour être juré est plus difficile à juger. Pour ce qui est des habitants ruraux, en 1830, le shérif de Québec considère qu'ils rencontrent les critères s'ils possèdent une maison et 10 à 12 acres de terres en culture,

15. La situation est légèrement embrouillée jusqu'en 1766. Une interprétation stricte de l'ordonnance de 1764 sur l'administration judiciaire veut que les jurés catholiques ne soient autorisés que dans le Banc du roi, mais dans les faits, les jurés catholiques sont aussi acceptés dans les Sessions trimestrielles depuis 1764-1765. Des instructions additionnelles au gouverneur mettent en doute l'interprétation stricte; enfin, une ordonnance de 1766 confirme le droit des catholiques d'être membres de tous les jurys, autant civils que criminels (4 George III, 17 septembre 1764, "An Ordinance for regulating and establishing the Courts of Judicature, Justices of the Peace, Quarter-Sessions, Bailiffs, and other Matters relative to the Distribution of Justice in this Province»; 6 George III, "An Ordinance to alter and amend an ordinance [...] passed the seventeenth day of September, $1764 », 1^{\text {er }}$ juillet 1766).

16. Y compris David David, qui est membre 16 fois entre 1796 et 1822.

17. 27 George III c.1 (1787). 
quoique son assistant déclare que c'est plutôt 40 arpents (environ 34 acres) en culture. En tout cas, la liste confectionnée en conséquence, qui se limite aux paroisses à 10 lieues ou moins de Québec, comprend environ 3800 noms, dans un territoire qui compte environ 10000 ménages, soit entre le tiers et la moitié. Pour la ville de Québec, sa liste comprend 1300 noms, pour environ 5000 ménages, soit environ le quart. Pour le district de Montréal, il n’y a aucune donnée comparable disponible pour les paroisses rurales, mais pour la ville, la liste des jurés potentiels de 1817-1818 comprend environ 1600 chefs de ménage, dont environ 1500 hommes, propriétaires ou locataires, qui rencontrent les critères de base. Cela représente environ la moitié des chefs de ménage; toutefois, en 1817, la population adulte mâle de la ville est d'environ 6000 personnes et c'est donc seulement le quart de cette population qui est éligible et, évidemment, environ le huitième de la population adulte ${ }^{18}$. Somme toute, dans le contexte colonial, les critères de sélection des jurés sont beaucoup plus larges qu'en Angleterre, encore que restrictifs.

Les différences avec l'Angleterre ne s'arrêtent pas aux critères de sélection. Le processus de sélection des jurys au Québec et au Bas-Canada est laissé presque entièrement entre les mains du shérif de chaque district judiciaire. Cette centralisation est d'autant plus surprenante que même si la machinerie d'administration locale anglaise (hundreds, connétables dans chaque paroisse, etc.) n'est pas reproduite au Québec et au Bas-Canada, il existe néanmoins des structures administratives locales analogues qui, en théorie au moins, auraient pu accomplir les mêmes tâches : notamment, les baillis élus dans chaque paroisse avant 1775 et les capitaines de milice qui les suivent ${ }^{19}$. De plus, tandis qu'en Angleterre les shérifs sont des notables nommés annuellement, au Québec et au Bas-Canada, ce sont des fonctionnaires permanents. Ce sont donc des officiers de l'État, nommés selon son bon plaisir par l'administration coloniale, qui contrôle la

18. P. J. R. King, “"Illiterate Plebeians, Easily Misled” », op. cit., 261 ; Fernand Ouellet, Le BasCanada, 1791-1840 : changements structuraux et crise (Ottawa, Éditions de l'Université d'Ottawa, 1976), 41-43; liste des jurys (ou rôle d'évaluation) de c.1817-1818, ANC RG4 B19 volume 1A; JCABC 39 (1830), Appendice SS ; Stephen Sewell (shérif de Québec) au secrétaire civil du gouverneur, ANC RG4 A1 volume 319-323, 14 mai 1830; recensement de 1831, dans JCABC 41 (18311832), Appendice OO. En 1817, la ville de Montréal compte entre 2800 et 3200 ménages (si l’on se fie à une population estimée à 16000 et à la taille moyenne des ménages en 1825 et 1831).

19. Donald Fyson, «La paroisse et l'administration étatique sous le Régime britannique (17641840)", dans Serge Courville et Normand Séguin, dir., Atlas historique du Québec : La paroisse (Sainte-Foy, Les Presses de l’Université Laval, 2001), 25-37. 
composition du Palladium of British liberty et la jouissance de la marque fondamentale de la citoyenneté britannique qu'est la participation aux jurys $^{20}$. Dans ce sens, les critiques des réformateurs dans les années 1820 sont tout à fait justes.

En soi, cette centralisation n'est qu'une innovation administrative, même souhaitable si l'on considère les problèmes du système anglais décentralisé. Mais elle laisse un pouvoir arbitraire aux shérifs et les procédures que ces derniers suivent en pratique vont souvent à l'encontre de la lettre de la loi et de l'esprit du système des jurys. Suivant la procédure dictée pour les jurys dans les causes civiles, les shérifs du district de Montréal, comme ceux des autres districts, créent des listes de chefs de ménage, à partir desquelles ils sélectionnent les jurés criminels ${ }^{21}$; toutefois, jusqu’aux changements apportés par la loi de 1832, ces listes sont des documents administratifs qui ne sont pas publics. Encore plus important, en sélectionnant des jurés, les shérifs semblent avoir appliqué trois critères additionnels qui ne sont pas fondés en droit positif.

D’abord, pour les jurys du Banc du Roi jusqu'en 1812, aussi bien grands que petits, et pour ceux des Sessions trimestrielles jusqu'à la fin des années 1820 , les shérifs les constituent presque exclusivement à partir des habitants de Montréal (qui ne représentent qu'environ $10 \%$ de la population $\mathrm{du}$ district), avec parfois quelques individus de la partie rurale de la paroisse de Montréal ${ }^{22}$. En 1817, le shérif, Frederick William Ermatinger, explique les raisons de cette pratique. L'absence de subdivisions administratives rend impraticable la constitution d'une liste de jurés qualifiés en dehors de la ville et ses environs, la plupart des ruraux étant en outre jugés trop ignorants pour servir comme jurés :

Q. Do you think it consistent with the liberty of British subjects, that the Trial by jury in the district of Montreal, in criminal cases, should be confined to the Citizens of the town and precincts of Montreal?

20. Le processus est décrit en détail dans JCABC 26 (1817), Appendice $\mathrm{K}$ et 39 (1830), Appendices EE et SS et dans deux lettres des shérifs de Québec (Sewell) et Montréal (Gugy) au secrétaire civil du gouverneur, ANC RG4 A1 volume 319-323, 14 mai 1830 et 17 mai 1830.

21. Pour le $\mathrm{xvIII}^{\mathrm{e}}$ siècle, nous avons deux listes pour le district de Montréal, l'une de 1765 (mais qui ne concerne que les Protestants : ANC RG4 A1, 5417-5419) et l'autre de 1785 (ANC RG4 A1, 9000-9007). Pour le début du xix ${ }^{\mathrm{e}}$ siècle, il y a trois listes, de 1811, 1813 et c.1817-1818 (ANC RG4 B19 volumes 1 et $1 \mathrm{~A}$; le contenu de la troisième liste suggère une date de 1817-1818 plutôt que 1816); celle de c.1817-1818 pourrait aussi être un rôle d'évaluation. Il y a aussi les listes produites en 1832-1835, en vertu de la loi de 1832 (ANC RG4 B19 volumes 2-6).

22. JCABC 26 (1817), Appendice K et 39 (1830), Appendice SS. 
A. I do. Instruction seems to be so little diffused, as I have already said, throughout the district at large, that I think those Laws better administered, and the liberty of the subject likely to be better protected, by taking jurors within those limits ${ }^{23}$.

Ermatinger explique toutefois qu'à partir de septembre 1812, il a commencé à recruter la moitié des grands jurés pour le Banc du Roi dans les paroisses rurales du district. Cela n'était pas effectué tant en réponse à des critiques de l'administration que par obéissance aux instructions du Gouverneur à la suite d'une représentation du grand jury de mars 1812 . Ce jury, à moitié britannique et sous la présidence du très tory James Caldwell, affirmait que, étendue à l'ensemble des habitants éligibles du district, l'obligation de servir comme grand juré permettrait à la population plus généralement de participer au système de justice criminelle anglais et donc d'apprécier ses bénéfices ${ }^{24}$. La pratique fut interrompue entre novembre 1822 et mai 1827, principalement en raison d'un manque de fonds pour convoquer les jurés des paroisses rurales ${ }^{25}$; pendant l'interruption, ce sont non seulement les membres de l'opposition politique qui demandent que les grands jurys soient constitués à partir du district au complet mais aussi, de façon répétée, les grands jurys eux-mêmes et le juge en chef du district, James Reid ${ }^{26}$. Mais même si la moitié des grands jurés du Banc du Roi proviennent des paroisses rurales, cela ne représente certainement pas la proportion réelle des ruraux parmi les personnes éligibles. La description fournie par Ermatinger des critères qu'il utilise pour choisir des jurés ruraux est évocatrice : «Those taken from the country are returned, after the strictest inquiry respecting their capacity to act as such, and their capability of bearing the expense of coming to town ${ }^{27}$. " Et tel que le graphique 1 le montre, quand la pratique reprend à partir de 1827 , c'est encore environ la moitié des grands jurés qui proviennent de la ville et cela, jusqu'au début des années $1830^{28}$.

\section{JCABC 26 (1817), Appendice K.}

24. ANQM TL30 S1 SS11, Registre de la Cour du Banc du Roi du district de Montréal, mars 1812.

25. Voir par exemple Uniacke à Cochran, 2 novembre 1822, dans ANC RG1 E15A volume 43 (1822) dossier «Miscellaneous Judicial 1822».

26. Montreal Herald, 17 mai 1823, 11 septembre 1824 et 12 mars 1825; Montreal Gazette, 12 mars 1825 .

27. JCABC 26 (1817), Appendice K.

28. Les analyses qui suivent sont basées sur une liste de 5793 grand jurés constituée à partir d'une variété de sources. Pour les Sessions trimestrielles il s'agit principalement des registres de la Cour entre 1779 et 1833 (ANQM TL32 S1 SS11). Pour le Banc du Roi et les sessions des Cours 
G RAPHIQ U E I

Résidence des grands jurés du Banc du Roi du district de Montréal, 1820-1833

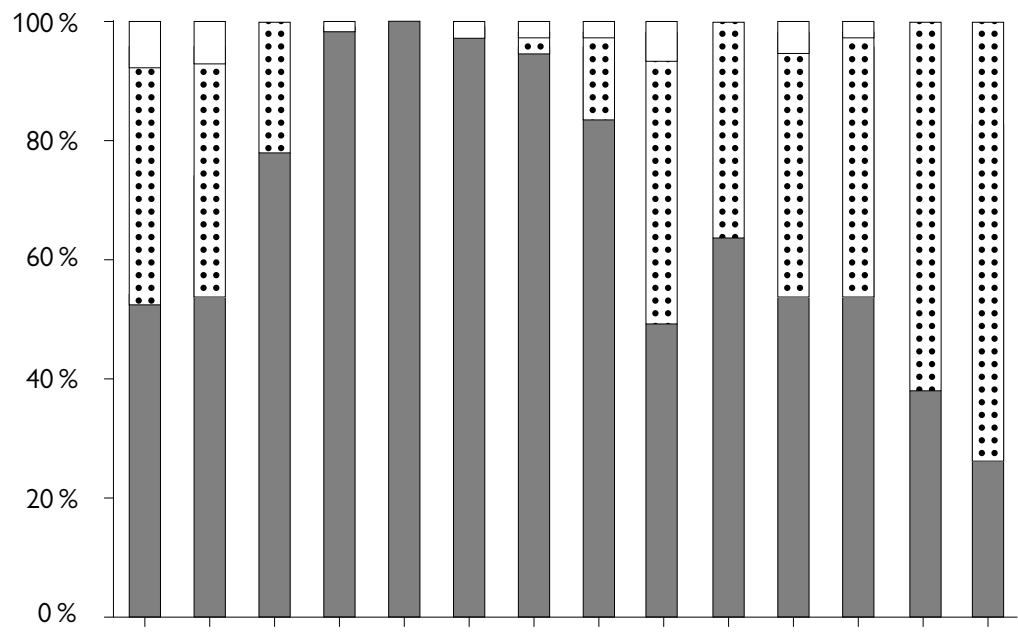

18201821182218231824182518261827182818291830183118321833

$\square$ Inconnue $\quad:::$ Ailleurs $\quad \square$ Montréal

GRAPHIQUE 2

Résidence des grands jurés des sessions trimestrielles du district de Montréal, 1820-1833

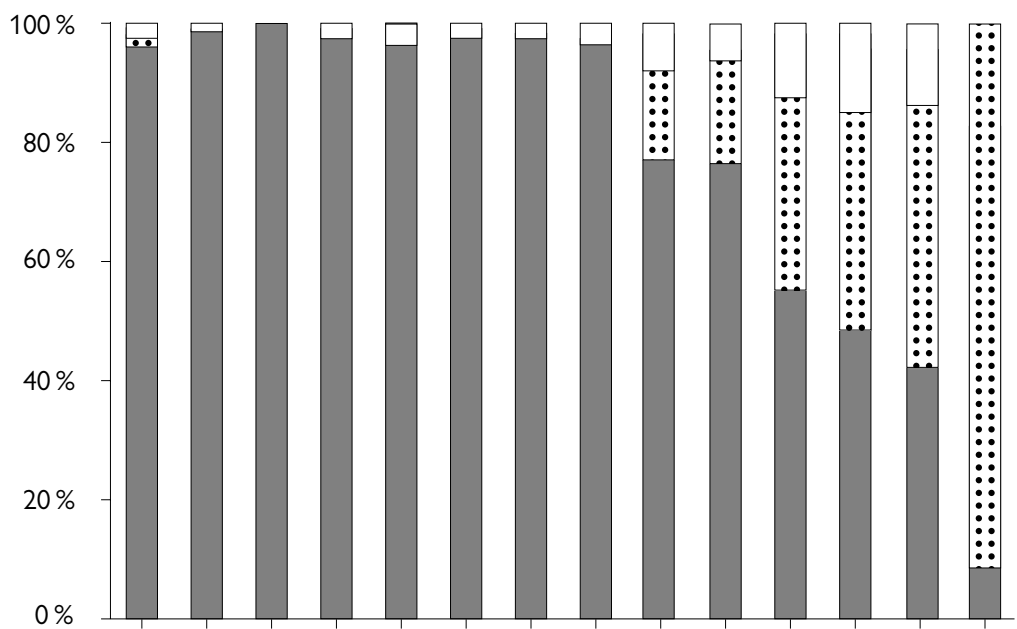

18201821182218231824182518261827182818291830183118321833

$\square$ Inconnue $\quad:::$ Ailleurs $\quad \square$ Montréal 
Enfin, même cet élargissement limité ne s'applique qu'aux grands jurys du Banc du Roi; ceux des Sessions trimestrielles (et les petits jurys des deux tribunaux) restent constitués très majoritairement de citadins jusqu'en 1830, malgré l'ajout de quelques ruraux (des paroisses de l'île de Montréal surtout) à partir de 1828, tel que le montre le graphique 2.

Ce n'est qu'à partir de 1830 que le shérif commence à élargir son bassin de recrutement de façon significative, ce qui a des effets majeurs sur la composition des jurys. Au sein des sessions trimestrielles en particulier, le nombre de citadins chute de façon dramatique entre 1829 et 1833 . Cela est particulièrement vrai après la mise en vigueur de la nouvelle loi sur les jurys, au milieu de 1832, qui transforme cette institution citadine en institution représentant un peu mieux l'ensemble du district ${ }^{29}$.

Un deuxième critère appliqué par les shérifs concerne les différents types de jurys. En droit anglais, il n'y a aucune distinction faite entre les critères d'admissibilité des grands et des petits jurés des différents tribunaux. Toutefois, en pratique, les grands jurés en Angleterre sont généralement choisis parmi un groupe d'élites plus exclusif que celui qui alimente les petits jurys. La même situation prévaut au Québec/Bas-Canada. Les listes de jurés potentiels qui ont survécu pour Montréal, notamment

d'Oyer et Terminer il s'agit d'un certain nombre de listes individuelles de jurés entre 1767 et 1794 (ANQQ TL18, S1, SS1 et TL999), de la liste de tous les jurés entre 1778 et 1784 (ANC RG4 A1, 8987-8996) et pour 1785 (ANC RG4 A1, 9266-9272), des registres pour 1802-1803 de même qu'entre 1812 et 1833 (ANQM TL30 S1 SS11) et de diverses représentations signées et publiées. Dans les analyses chiffrées, "Grands jurés» désigne des participations individuelles aux grands jurys; au total, il s'agit d'environ 1500 individus. L'analyse prosopographique des grands jurés (résidence et profession) est en outre fondée sur les listes des jurés potentiels de 1811, 1813 et 1832-1833 (ANC RG4 B19 volumes 1-6) et sur diverses autres sources prosopographiques, notamment les banques de données Parchemin : Banque de données notariales du Québec ancien (16351775) (Montréal, Société Archiv-Histo, 2000) et Thémis I : Cour du Banc du Roi en matière civile $d u$ district de Montréal (1791-1827) (Montréal, Société Archiv-Histo, 1999); Thomas Doige, An Alphabetical List of the Merchants, Traders and Housekeepers Residing in Montreal. The Second Edition (Montréal, 1820); j'ai également utilisé la version électronique produite par Robert C. H. Sweeny (St. John's, Memorial University of Newfoundland, 1997-2000); le Dictionnaire biographique du Canada; le recensement de 1825 pour Montréal, d'après la version électronique produite par Robert C. H. Sweeny, Manuscript Census Returns for Montréal, 1825 (St. John's, Memorial University of Newfoundland, 1997-2000; et Inventaires après décès du district judiciaire de Montréal, 1791-1840 (Montréal, Archives nationales du Québec, 1990).

29. Les «inconnus» au sein des grands jurés des sessions trimestrielles sont vraisemblablement très majoritairement des ruraux, compte tenu de la nature des sources utilisées pour les identifier : jusqu'au milieu de 1832, ces sources permettent l'identification plus systématique des individus urbains et ce n'est qu'à partir du milieu de 1832 que l'identification des ruraux devient beaucoup plus aisée, grâce aux listes de jurés potentiels de 1832-1833. 
celles de 1811 et 1813, semblent indiquer qu'en général, les shérifs ont inclus la plupart des chefs de ménage techniquement éligibles. Toutefois, les shérifs de Montréal appliquent une distinction évidente selon le type de jury et le tribunal. Les listes répartissent les chefs de ménage éligibles entre grands et petits jurys et, pour ce qui est de celle de 1813, entre ceux du Banc du Roi et des Sessions trimestrielles. La distinction semble se fonder sur la base de la valeur de la propriété, sur la profession et peut-être sur la connaissance personnelle du shérif, car ce ne sont que les plus fortunés qui sont désignés grands jurés potentiels ${ }^{30}$. Le résultat est clair : en 1813, dans une ville avec une population d'environ 13000 habitants et un district judiciaire d'environ 160000 individus, le shérif identifie 47 hommes, résidants de Montréal, comme grands jurés potentiels pour le Banc du Roi (auxquels s'ajoute un nombre égal de ruraux) et 96 pour les Sessions trimestrielles. La valeur annuelle moyenne de leurs propriétés se situe aux alentours de $£ 80$ pour le Banc du Roi et $£ 60$ pour les Sessions trimestrielles; en comparaison, même en 1825 et même en excluant les propriétés de petite valeur, seulement le quart des propriétaires de la ville ont des propriétés qui valent $£ 50$ ou plus ${ }^{31}$. Entre les mains des shérifs de Montréal, cette institution devient donc avant tout l'apanage des plus fortunés. Même pendant la période de 1812 à 1822, quand le shérif constitue les jurys du Banc du Roi à partir du district au complet, il applique des critères plus sévères quant au statut social des grands jurés. Ainsi qu'il le déclare en 1817, ces derniers, en particulier, devraient être "men of the first distinction in the county ${ }^{32}$ ». En effet, la moitié des grands jurés ruraux sont aussi des juges de paix, membres solides de la notabilité locale qui ne sont pas nécessairement des bureaucrates serviles, mais qui ne sont certainement pas, non plus, des opposants radicaux de l'ordre constitutionnel établi ${ }^{33}$.

30. La distinction entre les différents types de jurés est accentuée encore plus par le fait que le shérif pourrait ne pas avoir toujours respecté le cens de $£ 10$, car dans la liste de 1813 , un assez grand nombre de propriétaires dont la valeur annuelle de la propriété est inférieure à $£ 10$ sont indiqués comme éligibles pour le petit jury des Sessions trimestrielles. Ce problème reste à expliquer; un jugement définitif ne pourra cependant être posé qu'après une analyse des petits jurés réellement convoqués par le shérif.

31. Paul-André Linteau et Jean-Claude Robert, "Propriété foncière et société à Montréal : une hypothèse ", Revue d'histoire de l'Amérique française, 28,1 (juin 1974) : 55 ; leurs calculs, basés sur les rôles d'évaluation, excluent les propriétés d'une valeur annuelle de moins de $£ 5$ (voir page 49).

32. JCABC 26 (1817), Appendice K.

33. Sur environ 175 grands jurés ruraux entre 1812 et 1822,87 sont des juges de paix. 
TABLEAU I

\section{Proportion des grands jurésdu district de Montréal qui sont des Canadiens (en \%)}

\begin{tabular}{c|c|c}
\hline & Banc du Roi & Sessions trimestrielles \\
\hline $1767-1773$ & 50 & données manquantes \\
$1777-1787$ & 50 & 48 \\
$1787-1809$ & 49 & 53 \\
$1810-1829$ & 49 & 50 \\
$1830-1832 *$ & 53 & 61 \\
$1832-1833$ & 54 & 73 \\
\hline
\end{tabular}

* Avant la mise en vigueur de la loi de 1832.

Le troisième critère appliqué par les shérifs de Montréal est une innovation locale : dès le début du régime britannique, ils convoquent un nombre égal de grands jurés canadiens ${ }^{34}$ et britanniques. Ce ne sont pas toutes les personnes convoquées qui se présentent pour être assermentées, en raison de négligence et de dérogations, mais le tableau 1 montre que jusqu'à la fin des années 1820 , cela se reflète aussi dans la réalité. Ce n'est qu'avec les réformes du système de convocation des jurés du début des années 1830 que l'équilibre est rompu, en faveur des jurés canadiens, et cela surtout au sein des Sessions trimestrielles.

Cette pratique est une innovation significative par rapport à d'autres colonies où une minorité anglo-protestante fait face à une majorité étrangère. En Irlande, les catholiques sont exclus des grands jurys; à Singapour, ils sont composés de colons blancs avec quelques rares Chinois ${ }^{35}$. La pratique au Québec/Bas-Canada semble plutôt être une application novatrice du vieux principe du jury de mediate, par lequel les forains en Angleterre peuvent être jugés par un jury composé à moitié de leurs concitoyens $^{36}$. Le principe est stipulé pour les causes civiles dans la colo-

34. "Canadiens" désigne le groupe francophone de la population, quoique je sois conscient que le terme n'est pas parfait. La périodisation est fondée sur la préservation des sources et sur les changements juridiques ou administratifs.

35. Neal Graham, The Courts, Crime and the Criminal Law in Ireland, 1692-1760, op. cit., 119-132;

Y. K. Lee, "The Grand Jury in Early Singapore», op. cit.

36. Marianne Constable, The Law of the Other: The Mixed Jury and Changing Conceptions of Citizenship, Law, and Knowledge (Chicago, University of Chicago Press, 1994). 
nie dès $1766^{37}$, mais son application aux grands jurys (criminels) semble sans précédent et assure aux Canadiens une place importante dans cette institution, tout en refusant la notion d'une citoyenneté britannique fondée sur la religion telle qu'avancée par les grands jurés britanniques dans leur représentation de 1764 .

Les Canadiens, qui constituent environ $90 \%$ de la population du district, sont néanmoins numériquement sous-représentés sur les grands jurys. Certes, étant donné les critères de résidence urbaine et de place sociale élevée, cela ne signifie pas nécessairement, en soi, une sous-représentation de la population canadienne, en particulier durant les années 1820 , quand la population de la ville de Montréal, toutes classes confondues, approche les $50 \%$ de Britanniques. Mais la division égale entre jurés canadiens et britanniques devient foncièrement injustifiable quand les jurés du Banc du Roi sont constitués de l'ensemble du district. Dans leur ensemble, les deux autres critères ont des implications très claires pour la composition ethnique des jurys : la classe dans laquelle les Britanniques sont les plus fortement représentés, soit la bourgeoisie montréalaise, domine les grands jurys, même si on ne peut pas parler de domination britannique de l'institution. Tout indique que cette situation prévaut aussi dans l'autre district principal, le district de Québec ${ }^{38}$.

Par rapport à l'allégeance politique, la situation est moins nette que la rhétorique des journaux et des députés nationalistes le laisserait penser. Au $\mathrm{xVIII}^{\mathrm{e}}$ siècle, même si l'administration est dominée dans une grande mesure par le «French Party», l'exclusion des membres du "English Party», entre autres la grande majorité des marchands britanniques, reste impensable. Mais même si on ne se concentre que sur le Banc du Roi pendant la période de 1810 à 1829 , lors des affrontements fréquents entre Canadiens/ Patriotes et Tories, il n'y a certainement pas une exclusion systématique de jurés plus nationalistes ou une domination complète par les Tories. Parmi 320 grands jurés dont l’affiliation politique peut être iden-

37. 6 George III, «An Ordinance to alter and amend an ordinance $[\ldots]$ passed the seventeenth day of September, 1764» (1 juillet 1766).

38. Dans le district de Québec, on remarque le même biais urbain, sauf de façon temporaire pendant les années 1810 pour ce qui est des grands jurés du Banc du Roi (Gazette de Québec, 13 avril 1829, 10 septembre 1829 et 23 septembre 1835); il y a aussi la même sélection ethnique égale (comme en font foi les nombreuses listes des grands jurés préservées dans les dossiers du Banc du Roi de Québec, ANQQ TL18 S1 SS1). Le shérif de Québec semble néanmoins plus porté à modifier ses pratiques à partir de la fin des années 1820. La situation dans les plus petits districts judiciaires (Trois-Rivières, Gaspé et Saint-François) reste à explorer. 
tifiée, le plus grand nombre appartient certainement aux Tories, mais ils ne représentent que la moitié des jurés; les autres sont distribués à peu près également entre partisans plus radicaux du parti Canadien ou Patriote et réformateurs ou nationalistes modérés. Au sein des Sessions trimestrielles, c'est moins de $40 \%$ des jurés, dont l'affiliation politique peut être identifiée, qui se révèlent tories. En somme, comme c'est le cas pour beaucoup d'autres institutions locales, l'administration coloniale ne pratique pas une exclusion systématique même de ses opposants politiques; elle réserve plutôt ses machinations pour des causes politiques particulières.

Les effets des critères de sélection se voient aussi dans le statut social et la profession des grands jurés qui ont réellement siégé. Comme nous l'avons vu, le cens de $£ 10$ de valeur annuelle de propriété ou $£ 15$ de loyer, tout en restreignant l'accès aux jurys, pourrait tout de même laisser place à environ la moitié des chefs de ménage, ce qui comprendrait un bon

TABLEAU 2

Professions des grands jurés du Banc du Roi du district de Montréal ${ }^{\dagger}$

\begin{tabular}{|c|c|c|c|c|c|c|}
\hline & $\begin{array}{l}1767- \\
1773\end{array}$ & $\begin{array}{l}1777- \\
1787\end{array}$ & $\begin{array}{l}1787- \\
1809\end{array}$ & $\begin{array}{l}1810- \\
1829\end{array}$ & $\begin{array}{l}1830- \\
1832^{\text {t† }}\end{array}$ & $\begin{array}{l}1832- \\
1833\end{array}$ \\
\hline Nombre total de jurés & 181 & 558 & 173 & 917 & 78 & 63 \\
\hline Profession connue & $\begin{array}{c}177 \\
(98 \%)\end{array}$ & $\begin{array}{c}524 \\
(94 \%)\end{array}$ & $\begin{array}{c}173 \\
(100 \%)\end{array}$ & $\begin{array}{c}879 \\
(96 \%)\end{array}$ & $\begin{array}{c}73 \\
(94 \%)\end{array}$ & $\begin{array}{c}63 \\
(100 \%)\end{array}$ \\
\hline marchand & $86 \%$ & $89 \%$ & $79 \%$ & $62 \%$ & $47 \%$ & $17 \%$ \\
\hline marchand-propriétaire & $5 \%$ & $4 \%$ & $3 \%$ & $5 \%$ & $3 \%$ & - \\
\hline professionnel & $3 \%$ & $3 \%$ & $13 \%$ & $18 \%$ & $22 \%$ & $3 \%$ \\
\hline propriétaire $^{t t \dagger}$ & $3 \%$ & $1 \%$ & $1 \%$ & $7 \%$ & $11 \%$ & - \\
\hline élite indéterminée $\mathrm{ttt \dagger}^{t+1}$ & $4 \%$ & $2 \%$ & $3 \%$ & $4 \%$ & $8 \%$ & $3 \%$ \\
\hline boutiquier/commerçant & - & $<1 \%$ & $2 \%$ & $4 \%$ & $3 \%$ & $13 \%$ \\
\hline artisan & - & - & - & $<1 \%$ & $4 \%$ & $3 \%$ \\
\hline $\begin{array}{l}\text { fermier/habitant/ } \\
\text { cultivateur }\end{array}$ & - & - & - & - & $3 \%$ & $60 \%$ \\
\hline
\end{tabular}

† L'analyse est basée sur les identifications professionnelles dans les sources mentionées dans la note 28 . Évidemment, les catégories professionnelles sont imprécises, mais elles donnent une impression générale du statut social des jurés. J'utilise le terme «élite» dans un sens large, qui comprend à la fois la grande et la petite bourgeoisie, de même que les autres groupes élites comme propriétaires, seigneurs, etc.

t† Avant la mise en vigueur de la loi de 1832.

tt† Seigneur, rentier; les rares marchands-propriétaires sont assimilés aux marchands.

t†t† Gentilhomme, écuyer, bourgeois. 
nombre d'artisans et de cultivateurs. Les critères plus restrictifs du shérif par rapport au grand jury modifient substantiellement cette ouverture potentielle. Les tableaux 2 et 3 présentent les catégories professionnelles des grands jurés des deux tribunaux.

Avant 1830, les grands jurés des deux tribunaux sont donc presque entièrement des membres de la bourgeoisie. Ensemble, les artisans et les habitants composent moins de $5 \%$ des jurés aux Sessions trimestrielles de la paix et moins de $1 \%$ au Banc du Roi. De façon encore plus frappante, les grands jurys représentent en très grande majorité les intérêts commerciaux, même si on peut noter la montée de la classe professionnelle à partir du tournant du XIX ${ }^{\mathrm{e}}$ siècle. Presque entièrement absents sont les propriétaires, notablement les seigneurs. Leur absence des Sessions trimestrielles est compréhensible, compte tenu de leur rang. Par rapport au Banc du Roi, elle n'est pas expliquée par le biais urbain (plusieurs seigneurs demeurent en ville et leur absence persiste pendant la période où la moitié des grands jurés sont des ruraux) ni par une exemption automatique de la noblesse seigneuriale (compte tenu de la présence de quelques seigneurs). Les shérifs semblent tout simplement les avoir fréquemment exemptés de cette tâche civique particulière.

TABLEAU 3

Professions des grands jurés des Sessions trimestrielles du district de Montréal

\begin{tabular}{|c|c|c|c|c|c|}
\hline & $\begin{array}{l}1777- \\
1787\end{array}$ & $\begin{array}{l}1787- \\
1809\end{array}$ & $\begin{array}{l}1810- \\
1829\end{array}$ & $\begin{array}{l}1830- \\
1832^{\dagger}\end{array}$ & $\begin{array}{l}1832- \\
1833\end{array}$ \\
\hline Nombre total de jurés & 379 & 1508 & 1611 & 229 & 96 \\
\hline Profession connue & 339 (89 \%) & $1420(94 \%)$ & $1502(93 \%)$ & 184 (80\%) & $96(100 \%)$ \\
\hline marchand & $80 \%$ & $58 \%$ & $50 \%$ & $42 \%$ & $5 \%$ \\
\hline professionnel & $5 \%$ & $11 \%$ & $17 \%$ & $8 \%$ & $1 \%$ \\
\hline propriétaire ${ }^{t \dagger}$ & $1 \%$ & $2 \%$ & $<1 \%$ & $1 \%$ & - \\
\hline élite indéterminée ${ }^{\dagger \dagger \dagger}$ & $6 \%$ & $4 \%$ & $3 \%$ & $5 \%$ & $1 \%$ \\
\hline boutiquier/commerçant & $8 \%$ & $21 \%$ & $25 \%$ & $12 \%$ & $5 \%$ \\
\hline artisan & $1 \%$ & $3 \%$ & $3 \%$ & $13 \%$ & $4 \%$ \\
\hline $\begin{array}{l}\text { fermier/habitant/ } \\
\text { cultivateur }\end{array}$ & - & - & $1 \%$ & $18 \%$ & $83 \%$ \\
\hline
\end{tabular}

$\dagger \quad$ Avant la mise en vigueur de la loi de 1832.

t† Seigneur, rentier; les rares marchands-propriétaires sont assimilés aux marchands.

tt† Gentilhomme, écuyer, bourgeois. 
Au XviII ${ }^{\mathrm{e}}$ siècle, les grands jurys du Banc du Roi évoquent l'élite de la communauté marchande de la ville, aussi bien canadienne que britannique, avec des noms comme James McGill ou Jean-Baptiste Adhémar. Les grands jurés des Sessions trimestrielles semblent un peu moins composés de notables, quoique des noms comme Gabriel Franchère ou Alexander Henry évoquent encore cette même communauté marchande. À partir du tournant du $\mathrm{xIx}^{\mathrm{e}}$ siècle, les différences entre les grands jurys des deux tribunaux s'accentuent. D’abord, moins de grands jurés des Sessions trimestrielles portent l'étiquette plus prestigieuse de marchand. Même parmi les «marchands » des grands jurys du tribunal inférieur, très peu sont identifiables comme faisant partie de l'élite marchande de la ville. Par contre, les grands jurys du Banc du Roi gardent leur caractère exclusif : on y trouve encore des noms comme Austin Cuvillier, Peter McGill ou John Molson, tandis que, indicateur plus général de leur notabilité, près de $60 \%$ sont aussi juges de paix. Sous la pression des plaintes de la fin des années 1820 , le shérif semble modifier ses pratiques et commence à élargir un peu son bassin, mais ce n'est qu'avec la nouvelle loi de 1832, en vigueur à partir du milieu de l'année, que la domination commerciale et bourgeoise est définitivement renversée, remplacée par une domination nette de cultivateurs.

Dans leur composition sociale, les grands jurys du Banc du Roi avant 1832 suivent donc un modèle oligarchique anglais, plutôt que le modèle plus égalitaire proposé pour les États-Unis. Mais à la différence de la plupart des grands jurys anglais, ceux des Assizes des comtés, il y a cette prédominance écrasante de la classe marchande. En Angleterre, les grands jurys des Assizes rassemblent l'aristocratie rurale mais pas la classe marchande, qui est concentrée dans les villes. Même si en principe le Banc du Roi de Montréal est l'équivalent d'un Assize pour l'ensemble du district, ses grands jurys ressemblent plutôt à ceux des tribunaux urbains, tels ceux de Londres à la fin $\mathrm{du} \mathrm{XvII}^{\mathrm{e}}$ siècle ou de Halifax à la fin du $\mathrm{xvIII}^{\mathrm{e}}$, quoique avec un statut social encore plus élitiste. Quant aux grands jurys des Sessions trimestrielles, même en Angleterre ils comprennent un nombre significatif d'artisans et de petits fermiers; à Montréal, c’est plutôt la reproduction à plus petite échelle de l'emprise oligarchique qu'on voit dans le Banc du Roi ${ }^{39}$.

39. Sur l'Angleterre voir P. J. R. King, "“lliterate Plebeians, Easily Misled” », op. cit., 261-283; D. Hay, "The Class Composition of the Palladium of Liberty», op. cit., 329-348; J. M. Beattie, Crime and the Courts, 322-324 et "London Juries in the 1690s", dans J. S. Cockburn et T. A. Green, Twelve Men Good and True, op. cit., 243. Sur l'Amérique du Nord britannique et les États-Unis, voir Jim Phillips, «Halifax Juries in the Eighteenth Century », dans Greg T. Smith et al., dir., 
Un dernier élément notable de la composition des grands jurys de Montréal concerne la participation répétée des jurés. En Angleterre, la loi limite cette participation à une fois aux deux ou trois ans, quoique cette règle soit souvent transgressée. Le juré moyen aux Assizes du comté de Surrey, par exemple, est membre de trois jurys différents et le grand jury moyen, composé de 23 jurés, compte 15 jurés qui y ont déjà participé ${ }^{40}$. À Halifax à la fin du $\mathrm{xvIII}{ }^{\mathrm{e}}$ siècle, la participation répétée est encore plus fréquente, car les jurés sont convoqués pour une année entière à la fois ${ }^{41}$. Dans le district de Montréal, des jurés sont sommés de façon répétée à des séances successives. Donc, au Banc du Roi, le grand juré moyen participe à un peu plus de trois jurys, comme en Angleterre. La situation est semblable dans les Sessions trimestrielles, comme en fait foi l'adresse de James McGill, en tant que magistrat, au grand jury de la session de juillet 1794 : «Il y a tant parmi vous qui avez servi dans l'emploi respectable de Grands Jurés, et qui êtes si bien qualifiés pour remplir avec honneur les devoirs de cette charge importante, qu'il n'est guère nécessaire que la Cour emploie votre tems à vous les expliquer ${ }^{42}$. " Par surcroît, le même individu peut être appelé à participer au grand jury des deux tribunaux. Au $\mathrm{XVIII}^{\mathrm{e}}$ siècle, c'est souvent à la même époque; tandis qu'au XIX ${ }^{\mathrm{e}}$, c'est plutôt en succession, avec un grand juré des Sessions trimestrielles faisant le saut, plus tard dans sa vie, au grand jury du Banc du Roi. En tout, environ $40 \%$ des grands jurés du Banc du Roi ont déjà participé au grand jury des Sessions trimestrielles. Si l'on prend les deux tribunaux ensemble, jusqu'en 1830, le juré moyen est présent un peu plus de cinq fois; dans un grand jury moyen de 23 membres, 18 ont déjà l'expérience de cette tâche. Ce n'est qu'à partir du début des années 1830 que la situation change de façon radicale, avec l'élargissement des critères de sélection du shérif : parmi les grands jurés membres entre 1831 et 1833 , à peine le quart est expérimenté.

Pour ce qui est de l'administration de la justice, il est peut-être même souhaitable d'avoir des jurés expérimentés. Toutefois, le jury est plus qu'un élément de l'appareil judiciaire et ce service répété des jurés ne pouvait que réduire l'accès populaire, tel que le souligne La Minerve en 1828 :

Criminal Justice in the Old World and the New (Toronto, Centre for Criminology, University of Toronto, 1998); P. Romney, «From Constitutionalism to Legalism», op. cit., 130; R. D. Younger, The People's Panel, op. cit.; et C. Waldrep, "Egalitarianism in the Oligarchy", op. cit.

40. J. M. Beattie, Crime and the Courts, op. cit., 327-329; voir aussi D. Hay, "The Class Composition of the Palladium of Liberty ", op. cit., 343-348.

41. J. Phillips, «Halifax Juries in the Eighteenth Century », op. cit.

42. Gazette de Québec, 24 juillet 1794. 
Nous fréquentons depuis un grand nombre d'années les cours criminelles à Montréal; nous n'avons jamais vu un seul grand jury sans un, deux, trois ou même quatre membres de certaines familles, qui bien que très peu nombreuses et bien qu'égalées en respectabilité par un grand nombre d'autres, semblent en avoir acquis héréditairement le privilège. La famille tragique d'Atrée a fourni moins de sujets à la scène, que les familles Porteous et McKenzie n'ont fourni de grands jurés ${ }^{43}$.

Certains membres sont de véritables habitués; mais ce ne sont pas, comme le laisse sous-entendre La Minerve, des anglophones. Le record est détenu par Louis Plessis dit Bellair, un petit marchand de Montréal qui participe à 52 grands jurys des Sessions trimestrielles entre 1798 et 1829, soit à presque la moitié de toutes les sessions de la cour. Dix autres individus, tous Canadiens, sont membres d'au moins trente grands jurys. Encore plus frappant, les jurés canadiens sont présents en moyenne 5,5 fois, contre 4,5 fois pour les jurés britanniques. Même si nous limitons notre analyse au Banc du Roi pour la période 1812-1828, des dix jurés les plus réguliers, la moitié sont des Canadiens, la même proportion que la présence canadienne parmi les grand jurés. Pour ce qui est des Sessions trimestrielles, il y a une nette prédominance canadienne parmi les jurés les plus expérimentés : des vingt jurés qui participent le plus, il n’y a que deux Britanniques. Les membres les plus expérimentés de cette institution d'origine britannique sont des Canadiens.

Il est donc évident que la notion des grands jurys comme institution représentative, même issue de la bourgeoisie, subit des modifications significatives au Québec et au Bas-Canada. Les grands jurys sont représentatifs, mais d'un groupe en particulier, la bourgeoisie commerciale montréalaise. Comme La Minerve le déclare en 1827, «un juré [sic] [...] composé d’une classe de citoyens ou d'individus faisant partie du même ordre, est une atteinte évidente contre l'institution ${ }^{44}$ ». Mais cette "classe» n'est pas en réalité celle visée par le journal, ou par les jurés britanniques de 1764 ; telle que transposée aux grands jurys du district de Montréal entre 1764 et 1829, la reconnaissance de la citoyenneté était autant une question de classe, de profession et de résidence que d'ethnicité ou d'idéologie politique. 


\section{2 - LA PERCEPTION DES GRANDS JURYS AU SEIN DES ÉLITES}

La perception qu'avait La Minerve des grands jurys nous amène à notre deuxième questionnement, l'attitude des élites. La plupart des chercheurs sont d'accord pour affirmer que le rôle idéologique et politique du jury ne se transpose pas bien au contexte colonial et, en particulier, à la population canadienne. Ainsi, par rapport à la première décennie de l'administration civile britannique, Douglas Hay affirme que cette institution perd l'essentiel de sa signification, tandis que pour la période allant jusqu'à la Confédération, André Morel rejette l’idée que les jurys aient pu servir comme outil d'éducation juridique et constitutionnelle pour la population canadienne ${ }^{45}$.

Cette perspective résiste toutefois assez mal à un examen plus détaillé. Prenons les quelques décennies suivant la Conquête. Certes, il y a les commentaires négatifs de Michel Chartier de Lotbinière dans les années 1770, soulevés par Morel et Hay, quoiqu’ils visent surtout le rôle des jurys lors des procès. Par contre, la participation active des grands jurés canadiens dans l'élaboration de la représentation de 1764, de même que leur indignation subséquente, suggèrent une volonté dès le début d'utiliser ce nouvel outil d'expression. En effet, il n'y a aucune indication que les élites canadiennes aient entrepris un boycottage des jurys. Au contraire, même durant les années 1760, nous trouvons des grands jurés canadiens qui signent d'autres représentations, quoique avec la stipulation qu'ils le font après qu'une traduction leur a été lue ${ }^{46}$. Plus encore, en 1768 , le grand jury des Sessions trimestrielles de la ville de Québec fait une autre représentation, mais cette fois-ci entièrement en français et sous la présidence d'un Canadien ${ }^{47}$. Enfin, de façon plus générale, parmi les grands jurés des Sessions trimestrielles de Montréal mis à l'amende pour avoir négligé de se présenter, entre 1778 et 1830, seulement le tiers sont des Canadiens : les Canadiens prennent leurs devoirs plus au sérieux que leurs compatriotes britanniques ${ }^{48}$.

Les élites, autant canadiennes que britanniques, semblent convaincues de l'importance de l'institution, et des grands jurys en particulier, aussi y participent-elles volontiers. Cela dit, la vision que les élites en entretiennent varie énormément, comme le suggèrent les deux exemples présen-

45. D. Hay, «Meanings of the Law», op. cit., 94-97; A. Morel, «La réception du droit criminel anglais», op. cit., 521-523.

46. ANC RG4 A1, 4491-4492 (1765); Gazette de Québec, 11 août 1766.

47. ANQQ TL999, contenant 14, dossier «Banc du Roi 1764-1765-1769-1770-1774-1779».

48. ANQM TL32 S1 SS11. 
tés en introduction. Compte tenu de la grande variété au sein même des élites coloniales, il nous est impossible de rendre compte de chacune de leurs visions. Nous nous concentrerons plutôt sur l'attitude de deux groupes qui sont souvent dépeints comme étant aux antipodes idéologiques dans la colonie et cela, surtout à partir des années 1790 : d'une part, l'élite tory, surtout britannique; d'autre part, l'élite réformatrice, surtout canadienne, à tendance plutôt nationaliste.

L'attachement de l'élite tory, surtout britannique, au système des jurys est incontestable. Tel que l'énonce une lettre envoyée à la Gazette de Québec en 1793 : «Entre les perfections qui ont acquis la célébrité à notre Constitution, celle des Jurés est une des plus grandes ${ }^{49}$.» Par rapport aux grands jurys, l'attitude des Tories est peut-être la mieux exprimée dans les adresses (charges) aux grands jurys que livre le juge de la Cour du Banc du Roi ou le président des Sessions trimestrielles. Comme c'est aussi le cas en Angleterre, ces adresses permettent aux juges et aux magistrats, pour la plupart des partisans fermes de l'administration, non seulement d'instruire les membres dans leurs fonctions spécifiques, mais aussi d'esquisser une vision plus large des bénéfices des institutions britanniques. Dans ces adresses, le jury comme institution idéale figure souvent. Ainsi, dans l'une des premières adresses conservées, celle présentée au grand jury du Banc du Roi en 1797, le juge en chef Monk déclare que «the extensive powers vested with, and the great benefits to be expected from, a Grand Jury, are only experienced and felt by the people of a free Government». En 1810, Thomas McCord, président des Sessions trimestrielles, retrace les origines du grand jury jusqu'à l'époque saxonne et décrit son adoption à travers le monde pour démontrer qu'il est « the product of the highest wisdom, and the greatest benefit that has been conferred upon us by the mother country". Enfin, en 1829, David Ross, président des Sessions trimestrielles, fait un lien direct entre la structure de la justice criminelle, composée des juges, du grand jury et du petit jury, et la constitution politique, formée par le Roi, les Pairs et les Communes; une comparaison sans doute très flatteuse pour les boutiquiers et petits marchands qui composent le grand jury ${ }^{50}$.

Cet attachement à la place constitutionnelle idéale du grand jury le rend inconcevable, pour l'élite tory, comme institution de participation démocratique. La participation est toujours présentée comme un devoir, 
assumé de façon répétée par un groupe restreint d'individus. L'adresse du juge en chef Sewell au grand jury du Banc du Roi du district de Québec en 1808 exprime cela parfaitement : «Messieurs, nous sentons parfaitement, que les devoirs du Grand Juré, reviennent si fréquemment pour plusieurs Messieurs de ce District, que la tâche pourrait leur en paroitre un peu fatiguante à remplir; mais nous sommes convaincus qu'il n'y a point parmi vous qui regrettent le tems et la peine qu'ils y consacrent ${ }^{51}$.» À l'inverse, l'idée même d'un élargissement démocratique est sujette au ridicule. En 1833, peu après la mise en vigueur de la loi sur la qualification des jurés, la Montreal Gazette émet un commentaire satirique sur la composition du grand jury du Banc du Roi :

Independent of their almost total ignorance of reading and writing, not merely of the English but even of the French language, there is not in the very appearance and behaviour of the present Grand Jury, any thing to excite that reverence and respect, which every one acquainted with the Constitution has been taught to entertain for the Grand Inquest of the District [...] it is the first that has appeared in a uniform costume; hodden grey capot with horn buttons and sash. One man, in truly independent style, has doffed his capot, and sits in the box with only his vest of étoffe $d u$ pays, to which are attached drab barragon sleeves. The majority have their necks decorated with tri-color worsted comforters - in short, they are people well enough adapted for the Petty Jury, but completely out of their sphere in the capacity of Grand Jurors ${ }^{52}$.

Les Canadiens non instruits sont donc, à la limite, acceptables pour les petits jurys, mais certainement pas pour les grands; pour poursuivre la métaphore de Ross, les Communes ont envahi les Pairs.

Au-delà du grand jury comme symbole et comme institution participative, les élites tory préconisent aussi une fonction civique concrète pour l'institution. À une époque où les Tories sont constamment en minorité dans la Chambre d'Assemblée, le grand jury a le potentiel de jouer un rôle important comme institution représentative alternative. Le juge en chef Reid encourage activement la participation des grands jurys au débat politique. Non seulement répète-t-il les formulations standardisées qui somment les jurés de présenter "whatever evils and inconveniencies may be found to prevail $[\ldots]$ to the prejudice of the public interest», mais à plusieurs reprises il décrit aussi les grands jurés comme les représentants du district et les assure que «the voice and representation of a grand jury 
[...] will always meet with the consideration, not only from this court, but from every branch of this government, executive and legislative ${ }^{53}$ ».

Il y a toutefois des limites à mettre à cette participation politique. Ainsi, en novembre 1822, un grand jury de la Cour d'Oyer et Terminer - composé en grande partie de partisans torys - répond à l'appel de Reid avec une représentation remarquable. Se déclarant très conscients de leurs devoirs comme représentants du district, ils regrettent que la législature ait systématiquement ignoré les représentations de grands jurys antérieurs. Ils présentent alors une longue liste de doléances, dont les remèdes relèvent pour la plupart des compétences de la législature bas-canadienne : entre autres, la prolifération des loteries, la faiblesse des lois sur le revenu, la menace des contrefacteurs établis le long de la frontière américaine, l'état pitoyable des routes, le nombre considérable de médecins sans permis, en plus de nombreux commentaires défavorables sur l'administration de la justice criminelle. En mai 1823, le grand jury renchérit et critique le Conseil exécutif pour avoir refusé d'allouer un budget pour la sommation de témoins. Ces représentations semblent trop musclées même pour Reid. En 1823, dans son adresse, il signale que les représentations «must be directed to objects properly within your cognizance, and not extend to those authorities or departments, over which, neither the grand jury, nor the Court have any jurisdiction or controul $[s i c]^{54}$ ». C'est un renversement radical pour un juge qui, jusque-là, prônait activement un rôle politique élargi pour le grand jury.

Les attitudes de l'élite réformatrice, notamment celle qui gravite autour de formations politiques nationalistes comme les partis Canadien et Patriote, sont plus complexes. Jusqu'aux années 1820, les commentaires sur les jurys sont assez rares, mais il est clair que les élites canadiennes restent convaincues de la nécessité des jurys en général et de l’importance particulière du grand jury pour l'intérêt commun. Déjà en 1790, Fleury Mesplet, dans la Gazette de Montréal, déclare que «Les soins \& l'attention de Mrs. les grands Jurés à dévoiler $\&$ dénoncer tout ce qui attaque $\&$ blesse les droits $\&$ la sûreté de la Communauté $\&$ des individus, méritent notre reconnoissance $\&$ un remerciement public» et les nomme «les vrais patriotes qui sacrifient le soin de leurs intérêts personnels à celui de la culte publique ${ }^{55}$ ». Les commentaires sur les grands jurys continuent sporadiquement par la suite,

53. Ibid., 16 mai 1821.

54. Ibid., 6 septembre 1823.

55. Ibid., 6 septembre 1823; "Report of a Committee of the Whole Council... ", ANC RG4 A1, volume 220-224, 9 août 1823. 
mais à partir de la fin des années 1820 , l'élite réformatrice commence à se servir plus systématiquement de la question des jurys pour attaquer leurs opposants autocratiques et les examens critiques du système en place se multiplient, dans la Chambre d'Assemblée aussi bien que dans des journaux comme La Minerve ou Le Canadien.

Certains ont postulé qu’à partir des années 1820, le mouvement Patriote en général commence à se tourner de plus en plus vers des formes politiques républicaines et américaines ${ }^{56}$. Les attitudes des élites réformatrices nous obligent à nuancer cette affirmation. Tout comme aux États-Unis, le jury, en tant qu'institution dotée d'un long héritage britannique, n'est jamais remis en cause; plutôt, les élites réformatrices s'approprient l'héritage britannique que l'administration est accusée de bafouer. Dans le cadre des travaux du comité de la Chambre d'Assemblée de 1829 sur la qualification et la sommation des grands et petits jurés, le discours du porte-parole du comité, le Patriote modéré (et futur juge) Vallières de Saint-Réal, est très évocateur: L'épreuve par jury ne fut pas connu $[s i c]$ de nos ancêtres canadiens [...] Les Anglais qui non seulement ont conservé l'épreuve par jury, mais qui, la considérant avec raison comme un de leurs droits les plus essentiels, se sont attachés à perfectionner cette institution salutaire, l'introduisant en Canada, avec le reste de leurs lois criminelles, aussitôt après qu'ils eurent fait l'acquisition de ce pays. Ainsi, les habitans du Canada, en devenant sujets britanniques, se sont trouvés revêtus du privilége [sic] inestimable qui met la vie, la liberté et les biens de chaque citoyen sous la garde de son pays, et qui défend d'y porter atteinte sans l'avis et le jugement de douze hommes libres et loyaux (liberi et legales) pris dans son voisinage ${ }^{57}$.

Par contre, suivant l'influence du républicanisme français et américain et comme c'était le cas pour le parlementarisme ${ }^{58}$, l'élite réformatrice met surtout l'accent sur les droits et la liberté, non seulement de l'accusé mais aussi des jurés. Déjà en 1812, l'Assemblée législative forme un comité pour examiner les qualifications et la sélection des grands et des petits jurés, en dépit de l'opposition des membres tory. En 1817, à la suite des plaintes sur la sélection des grands jurys par le shérif du district de Montréal, un autre

56. Louis-Georges Harvey, «Le mouvement patriote comme projet de rupture (1805-1837)», dans Yvan Lamonde et Gérard Bouchard, dir., Québécois et Américains : la culture québécoise aux $X_{X}{ }^{e}$ et $X X^{e}$ siècles (Saint-Laurent, Fides, 1995), 87-112.

57. JCABC 38 (1829), 336.

58. L.-G. Harvey, «Le mouvement patriote», op. cit.; Jean-Pierre Wallot, «La pensée révolutionnaire et réformiste dans le Bas-Canada (1773-1815)", dans Un Québec qui bougeait : trame sociopolitique au tournant du XIX ${ }^{e}$ siècle (Montréal, Boréal, 1973), 253-318. 
comité déclare que «tout homme [qui satisfait aux conditions de la loi] a droit d'être juré dans les affaires criminelles de cette province ${ }^{59}$ ». En 1830, le comité spécial de la Chambre d'Assemblée va encore plus loin en déclarant que «le droit des citoyens d'être jurés et celui d'un peuple d'élire et de donner son avis sur les lois par les représentants de son choix et tirés de son sein, sont de pair et ne peuvent exister l'un sans l'autre» et préconise que les jurés (sans distinguer entre les grands et les petits) «doivent être pris à même la masse du peuple ${ }^{60}$ ». Le contraste avec l'adresse de Ross au grand jury des Sessions trimestrielles est frappant. Ross évoque la division tripartite classique du Roi (les juges), des Pairs (les grands jurés) et des Communes (les petits jurés), faisant ainsi appel à la division classique du pouvoir dans le système constitutionnel anglais. Les membres du comité spécial, par contre, en faisant une équivalence entre la participation aux jurés et la démocratie, font appel plutôt aux notions de démocratie directe d'inspiration plus républicaine.

Le discours des élites réformatrices s'adresse toutefois au système des jurys en général et vise uniquement le rôle du jury lors du procès criminel. Le droit essentiel que ces élites défendent est l’idéal du jury, ce «droit de naissance [...] qui appartient à tout sujet Anglois, d'être jugé par ses pairs et de les juger", «l'apanage essentiel de la liberté dans notre gouvernement $[\ldots]$ impossible d'en séparer notre constitution sans le détruire ${ }^{61}$ ». Les critiques des réformateurs visent la sélection et la composition des deux types de jurys et le discours démocratique semble appliqué aux deux, parfois presque indifféremment : par exemple, en 1836, dans un article sur les grands jurys de Montréal, La Minerve défend la participation de grands jurés illettrés, entres autres en déclarant que les jurys (en général) sont le «boulevard des libertés d'un peuple ${ }^{62}$ ». Par ailleurs, dans cette conception du jury, il n'est jamais question d'une fonction explicite pour le grand jury dans le processus politique, comme corps représentatif du district. L'élite réformatrice contrôle déjà l'institution démocratique de son choix, la Chambre d'Assemblée, et ses confrontations avec l'administration et les partisans tory concernent essentiellement le pouvoir de cette institution. Dans le processus politique tel que le conçoivent les élites réformatrices, le grand jury est une institution superflue.

59. JCABC 20 (1812), 110; 26 (1817), 494 et Appendice K. [Nos italiques]

60. JCABC 39 (1830), Appendice SS.

61. La Minerve, 13 août 1827,2 juillet 1827 .

62. Cité dans Le Canadien, 4 novembre 1836. 
Enfin, même si les droits démocratiques et la liberté sont au cour des demandes de l'élite réformatrice pour une modification du processus de sélection des jurys, leur discours prend aussi souvent une tournure ethnolinguistique. Ainsi, en 1827, La Minerve déclare :

Un grand nombre des Jurés de ces villes d'abord est composé d'hommes quelquefois entièrement étrangers aux accusés dont la cause est portée devant eux. Ils le sont sur tout ce qui tient aux habitudes et aux mœurs, à l'éducation, à la langue, aux usages, au pays, à la profession, $\& c$. ... Souvent ils sont nés dans un autre hémisphère, ou s'ils ont pris naissance sur la partie du globe que nous habitons, la différence sous d'autres rapports n'est pas toujours moins marquée ${ }^{63}$. En 1829, Vallières de Saint-Réal déclare, par rapport à l'ordonnance de 1787 qui élargissait la qualification des jurés aux locataires :

[...] votre Comité ne peut voir dans la loi provinciale sous considération, que l'expression du désir de la part des hommes en autorité, d'éloigner les propriétaires Canadiens ou de les ravaler en leur donnant pour égaux, dans l'honorable et importante fonction des jurys et enquêtes, des hommes sans facultés et dénués d'indépendance, au risque d'abâtardir cette belle institution des Jurys, en vailissant les jureurs ${ }^{64}$.

Il est alors aisé de distinguer deux concepts très différents du grand jury. D'une part, une institution digne de Burke, source du bon ordre et de la stabilité constitutionnelle, l'apanage des classes supérieures, à ne pas mettre entre les mains du peuple vulgaire et non instruit; d'autre part, selon une conception plus républicaine, un avatar de la démocratie et du droit du peuple. D’un côté, une partie essentielle du processus politique; de l'autre, une institution purement juridique.

Mais il est aussi essentiel de ne pas exagérer ces différences. En particulier, la conception «démocratique» appuyée par les élites réformatrices se fonde encore sur une démocratisation limitée; beaucoup plus limitée que la franchise, par exemple. Les élites réformatrices ne s'opposent pas à la qualification restrictive des jurys, mais plutôt au fait que tous les habitants qualifiés ne sont pas inclus. De plus, il n'est jamais question d'inclure la participation des femmes aux jurys; comme pour le droit de vote, la participation civique reste une affaire masculine ${ }^{65}$. La loi de 1832, œuvre des réformateurs qui passe presque sans débat, augmente plutôt que de

63. La Minerve, 16 août 1827.

64. JCABC 38( 1829), 343.

65. Voir à ce sujet Allan Greer, "La république des hommes : les Patriotes de 1837 face aux femmes ", Revue d'histoire de l'Amérique française, 44,4 (printemps 1991) : 507-528. 
diminuer la qualification pour être juré, en particulier pour les grands jurys : pour le Banc du Roi, elle atteint $£ 25$ de valeur annuelle d'une tenure en freehold ou $£ 60$ par année en loyer, tandis que pour les sessions trimestrielles, c'est $£ 15$ de valeur annuelle d'une tenure en freehold ou $£ 40$ par année en loyer. Les femmes ne sont pas expressément exclues par la loi, mais cette exclusion semble implicitement comprise, car les listes dressées selon la loi ne contiennent aucune femme ${ }^{66}$. Il y a donc beaucoup plus une adoption formelle de la tradition anglaise qu'une démocratisation à l'américaine. Cet acte semble satisfaire les élites réformatrices. Un an plus tard, en 1833, James Stuart, le solliciteur général réformiste, propose des modifications substantielles, entre autres que la qualification pour les petits jurés soit abaissée à celle des électeurs et que les grands jurys soient membres pour trois années à la fois; les députés Patriotes, y compris Papineau, s'opposent à tout changement à une loi jugée sans défauts ${ }^{67}$. En effet, dans les 92 résolutions de 1834, qui expriment pourtant des critiques sévères et détaillées à l'endroit de l'administration coloniale en général, y compris des critiques détaillées visant l'administration de la justice, il n'est aucunement question des jurys. Loin de vouloir remplacer cette institution britannique, les élites réformatrices cherchent à la revaloriser en retournant à ses racines anglaises profondes. Tel que le déclare La Minerve en 1833, le jury est «le plus bel appanage [sic] d'un sujet britannique et que nous revendiquons avec orgueil, nous qui sommes descendus de parens français mais qui savons apprécier les avantages attachés à notre condition de sujets anglais ${ }^{68}$ ".

\section{3 - LE GRAND JURY DANS LE PROCESSUS POLITIQUE}

Au-delà de sa composition et de la perception des élites, quel est le rôle réel du grand jury dans le processus politique? C'est surtout par voie de ses représentations que le grand jury peut participer au processus politique; tel que le déclare Austin Cuvillier, président des Sessions trimestrielles, en 1836, «une partie importante de leur charge [consiste] à faire des représentations au gouvernement sur l'administration des affaires locales et publiques. C’est l'exercice zélé et consciencieux de ces devoirs qui donne tant de poids aux représentations des grands jurés, qui sont généralement regardés comme des émanations de l’opinion publique.» Le processus est

66. 2 William IV c.22 (25 février 1832); ANC RG4 B19 volumes 2-6.

67. Gazette de Québec, 15 décembre 1832, 31 décembre 1832, 9 janvier 1833 et 30 janvier 1833. 68. 4 mars 1833. 
assez simple et ressemble à une pétition collective. Le grand jury dresse une représentation, le plus souvent par écrit, adressée à la Cour, qui identifie un ou plusieurs problèmes à résoudre soit par la Cour, soit par les autres niveaux de gouvernement. Selon un observateur européen écrivant en 1833, en raison même de cette action du grand jury : «Les assises canadiennes constituent, pour ainsi dire, une représentation de district ${ }^{69}$. . Mais concrètement, quel rôle politique jouaient ces représentations? Quelques observations brèves sur les représentations des grands jurys du district de Montréal nous permettent d'avancer un certain nombre d'hypothèses.

D'abord, il importe de distinguer deux niveaux d'activités politique, coloniale et locale, car l'importance du rôle politique des grands jurys varie considérablement selon ces différents éléments. Au niveau de la politique coloniale, leur rôle est relativement limité. Le premier grand jury des sessions trimestrielles de Québec tente de se transformer en institution représentative, au point de rester constitué même après la fin des Sessions trimestrielles et d'inviter la population à lui adresser ses doléances ${ }^{70}$, mais cette tentative échoue. En général, les grands jurys font relativement peu de représentations en rapport avec la politique coloniale. Ainsi, dans les archives du Secrétaire civil du gouverneur entre 1764 et 1792, la source principale des représentations et pétitions adressées à l'administration coloniale avant la mise en place de l'Assemblée législative, j'ai identifié moins d'une dizaine de représentations de grands jurys de toute la colonie ${ }^{71}$. À partir du $\mathrm{XIX}^{\mathrm{e}}$ siècle, ce nombre augmente à environ une cinquantaine entre 1800 et 1830 pour tous les districts, dont une vingtaine pour celui de Montréal. La plupart ont tout de même un créneau très spécifique : la justice criminelle en tant que telle. Comme l'a démontré Jean-Marie Fecteau, les grands jurys du Banc du Roi en particulier ajoutent leurs voix au mouvement plus large parmi les élites pour une réforme du système ${ }^{72}$. Outre ces représentations, les grands jurys du district de Montréal se prononcent de temps à autre sur d'autres aspects de l'administration coloniale, mais ce sont des exceptions. Par exemple, dans le débat quasi constant autour du système de justice civile, il n'y a qu'une seule représentation du district de Montréal, en 1828, qui

69. Isidore Lebrun, Tableau statistique et politique des deux Canadas (Paris, Treuttel et Würtz, 1833), 445.

70. Gazette de Québec, 22 novembre 1764.

71. ANC RG4 A1. Évidemment, cela dépend également de la préservation des documents.

72. Jean-Marie Fecteau, La pauvreté, le crime, L'État au Québec, de la fin du XVIII siècle à 1840 (Montréal, VLB, 1989), passim. 
l'aborde. Au niveau colonial, le rôle politique du grand jury reste surtout lié à sa participation à des causes politiques.

Cette fréquence relativement faible des interventions des grands jurys au niveau colonial est parfaitement compréhensible. D’autres moyens de pression plus efficaces existent, notamment les pétitions (adressées à la fois à l'administration coloniale et à l'Assemblée), les journaux et, à partir de 1792, l'Assemblée elle-même. De plus, sauf si les représentations sont publiées ou transmises au gouvernement central, les grands jurés, ceux de Montréal du moins, ne s'adressent pas nécessairement à des joueurs clés de la politique coloniale : le juge en chef du Banc du Roi (dont les interventions dans l'administration sont surtout limitées au droit et à la justice, en particulier sous Reid) ou les magistrats locaux.

Au niveau local, par contre, la situation est très différente. Dans les villes, il n'y a aucun gouvernement représentatif, l'administration municipale étant sous le contrôle des juges de paix urbains. Ces mêmes juges de paix sont membres du banc des Sessions trimestrielles et reçoivent les représentations des grands jurys qui ont donc un accès direct aux responsables de l'administration locale ${ }^{73}$. À ce niveau, les grands jurys des Sessions trimestrielles, en particulier, jouent un rôle significatif dans le processus politique, en donnant à la bourgeoisie locale un moyen de commenter et d'influencer l'exercice du pouvoir local. Et ils ne manquent pas cette occasion : pour Montréal, entre 1765 et 1832, les grands jurys des Sessions trimestrielles font au moins 83 représentations concernant des questions publiques, dont la vaste majorité (73) visent l'administration locale de la ville. Tel que l'indique le tableau 4, dans ces représentations, les grands jurys font quelque 204 recommandations distinctes, qui couvrent une vaste gamme de sujets.

Le tableau 4 montre bien les intérêts variés des élites d'une petite ville coloniale; il montre aussi une croissance importante dans le nombre de recommandations au $\mathrm{XIX}^{\mathrm{e}}$ siècle. Un examen plus précis de certaines recommandations décrit encore plus clairement les désirs des marchands et des commerçants de la ville. Outre l'accent considérable mis sur le développement de l'environnement bâti, ce sont aussi les colporteurs, les ta-

73. Pour Montréal, voir Donald Fyson, «Les structures étatiques locales à Montréal au début du xix ${ }^{\mathrm{e}}$ siècle ", Les Cahiers d'histoire, 17,1-2 (1997) : 55-75. Pour Québec, l'administration municipale d'avant 1840 est abordée dans Antonio Drolet, La Ville de Québec : histoire municipale, 2 : Régime anglais jusqu'à l'incorporation (1759-1833) (Québec, Société historique de Québec, 1965) et dans David-Thiery Ruddel, Québec, 1765-1832 : l'évolution d'une ville coloniale (Hull, Musée canadien des civilisations, 1991). 
TABLEAU 4

Recommandations des grands jurys des Sessions trimestrielles de Montréal concernant l'administration locale, 1765-1832 ${ }^{\dagger}$

\begin{tabular}{l|c|c|c}
\hline & $\mathbf{1 7 6 5 - 1 7 9 1}$ & $\mathbf{1 7 9 2 - 1 8 0 9}$ & $\mathbf{1 8 1 0 - 1 8 3 2}$ \\
\hline $\begin{array}{l}\text { Représentations concernant } \\
\text { le public }\end{array}$ & 22 & 15 & 46 \\
$\begin{array}{l}\text { Recommandations concernant } \\
\text { l'administration locale }\end{array}$ & 49 & 26 & 129 \\
Recommandations concernant : & & & \\
l'environnement bâti et la voirie & $25(51 \%)$ & $14(54 \%)$ & $42(33 \%)$ \\
le feu & $4(8 \%)$ & $2(8 \%)$ & $5(4 \%)$ \\
l'approvisionnement & $1(2 \%)$ & $1(4 \%)$ & $22(17 \%)$ \\
la santé et la sécurité physique & $6(12 \%)$ & $2(8 \%)$ & $34(26 \%)$ \\
l'assistance sociale & $3(6 \%)$ & $1(4 \%)$ & $10(8 \%)$ \\
la moralité & $4(8 \%)$ & $6(23 \%)$ & $16(12 \%)$ \\
le crime, la justice et la police & $14(29 \%)$ & $6(23 \%)$ & $23(18 \%)$ \\
l'enfermement & $1 \dagger$ & - & $17(13 \%)$ \\
divers & $3(6 \%)$ & - & $2(2 \%)$ \\
\hline
\end{tabular}

$\dagger \quad$ Ces représentations proviennent surtout des registres des Sessions trimestrielles (ANQM TL32 SI SSII) avec quelques représentations additionnelles des dossiers des Sessions (ANQM TL32 SI SSI) et dans les journaux. Une recommandation peut concerner plus d'un type de sujet.

t† Inclut la Maison de correction mais pas la prison commune, qui n'est pas sous contrôle local.

verniers sans permis, les charretiers insubordonnés et autres groupes d'individus nuisibles à la solde de ces hommes qui reçoivent une attention particulière $^{74}$. À partir des années 1810 , les représentations font également état de ce souci constant pour le désordre urbain alors en vogue : un grand nombre de représentations vise la santé publique et c'est une représentation du grand jury des Sessions trimestrielles en 1817, transmise d'abord au Gouverneur et ensuite à l'Assemblée, qui mène à l'établissement du guet de la ville ${ }^{75}$. La vision morale de cette classe est aussi manifeste. Les tavernes et les maisons de débauche sont des cibles fréquentes : en 1823, par exemple, une représentation qualifie ces dernières «the greatest evil

74. Entre autres, ANQM TL32 S1 SS11 9 octobre 1766, 12 avril 1788, juillet 1794, 29 octobre 1814, 29 octobre 1825, 30 octobre 1828, 30 avril 1830; Montreal Herald, 25 novembre 1820, 17 septembre 1823 ; Montreal Gazette, 23 novembre 1822, 15 septembre 1828.

75. JCABC 27 (1818), 76-79. 
to which society falls a prey [...] the receptacles of all that is criminal» et de dangereuses pour «the morals of the rising generation ${ }^{76}$ ». Mais notons aussi le souci pour les pauvres "méritants", comme dans cette représentation de 1814 qui condamne la spéculation affectant le bois de chauffage "qui pourroit devenir très dangereux voyant que le pauvre peuple est incapable de se pourvoir de bois de chauffage au moyen de cette spéculation qui pourroit induire le pauvre peuple a des conséquences très funestes»; ou encore dans la condamnation des pauvres non méritants, comme dans une représentation de 1821 contre la tolérance des mendiants qui les distingue clairement des "vrais pauvres incapables de gagner leur vie ${ }^{77}$ ».

Au-delà de leurs objets, les représentations des grands jurys sont parfois utilisées pour critiquer l'administration municipale des juges de paix et leurs officiers inférieurs. Ainsi, en 1788, un grand jury des Sessions trimestrielles se plaint de la négligence de l'inspecteur de police; en 1821, un autre condamne fortement les juges de paix pour apathie et inefficacité; et encore plus directement, un grand jury de la Cour d'Oyer et Terminer en 1820 - jury qui comprend trois juges de paix membres de l'administration municipale - après avoir critiqué sévèrement cette administration, demande l'incorporation de la ville ${ }^{78}$. Dans le contexte d'un gouvernement non élu, le grand jury est un des moyens clés pour les citoyens les mieux nantis d'exprimer leur désapprobation.

De plus, il semble que certains citadins perçoivent le grand jury des Sessions trimestrielles comme un corps qui pourrait les représenter auprès des magistrats. Ainsi, les grands jurys, de temps à autre, reçoivent directement des pétitions de citoyens : en 1825, par exemple, une pétition de plus de cent résidants se plaignant de l'administration municipale est envoyée aux grands jurys plutôt qu'aux magistrats ${ }^{79}$. À un autre niveau, même la législature donne un rôle représentatif au grand jury des Sessions trimestrielles dans l'administration municipale, en lui confiant un contrôle sur la nomination des assesseurs qui déterminent les impôts fonciers à partir de 1796, des directeurs de la maison d'industrie à partir de 1818 et des membres de la société de feu à partir de $1829^{80}$.

76. ANQM TL32 S1 SS11, 19 juillet 1823.

77. ANQM TL32 S1 SS11, 29 octobre 1814, 19 janvier 1821.

78. ANQM TL32 S1 SS11, 24 janvier 1788, 19 juillet 1821; Montreal Gazette, 29 novembre 1820, 11 septembre 1824, 12 mars 1825 .

79. ANQM TL32 S1 SS11, 19 juillet 1825 ; la pétition originale est dans ANQM TL32 S1 SS1, 15 juillet 1825 .

80. 36 George III c.9 (1796); 58 George III c.15 (1818); 9 George IV c.16 (1829); 9 George IV c.57 (1829). 
Enfin, le rôle politique des grands jurys à un niveau local, à la différence de sa composition ou de son rôle dans les causes criminelles, ne semble pas avoir été une source importante de conflit entre les élites canadiennes et britanniques. Presque toutes les représentations des Sessions trimestrielles sont signées à la fois par des jurés canadiens et britanniques et sont rédigés parfois en français, parfois en anglais. Les raisons sont évidentes : en matière d'administration locale, les élites locales qui dominent les grands jurys partagent des valeurs communes.

Un dernier point concerne les effets concrets de ces représentations : les magistrats en tiennent-ils compte? Dans un certain nombre de cas, les représentations mènent directement à la rédaction de nouveaux règlements municipaux par les magistrats : c'est le cas pour les représentations concernant le bois de chauffage et les mendiants, mentionnées ci-dessus. Les grands jurys jouent donc un rôle similaire aux consultations publiques dans le processus législatif normal; un règlement municipal de 1810 qui interdit les inhumations dans la ville (ce qui deviendra plus tard une source de discorde entre les magistrats et la hiérarchie catholique) cherche même à légitimer son contenu en commençant par : «Un des précédents Grands Jurés ayant représenté comme une nuisance, les enterrements dans la ville $^{81}$...» Dans un même ordre d'idées, en 1786 les magistrats de la ville justifient leurs dépenses pour paver le marché en invoquant une recommandation du grand jury ${ }^{82}$. Si elles touchent des matières en dehors des compétences des magistrats, les représentations peuvent être acheminées à l'administration centrale; ainsi, c'est une représentation des Sessions trimestrielles en janvier 1791, envoyée au gouverneur, qui mène éventuellement à la démolition des fortifications de la ville, étape fondamentale pour l'expansion urbaine ${ }^{83}$. Néanmoins, le grand jury n'a aucun pouvoir coercitif et ses représentations sont assez fréquemment ignorées. Cela devient progressivement le cas dans la deuxième moitié des années 1820, sous l'administration de juges de paix tories plus autocratiques comme Samuel Gale et David Ross; en 1831, par exemple, le grand jury des Sessions trimestrielles se plaint que «in times past the recommendations of one Grand Jury have been generally forgotten by the members of the Court during the next term ${ }^{84} »$. 
Tout de même, c'est à ce niveau local, et en grande partie au sein des Sessions trimestrielles, que les grands jurys jouent un rôle essentiel dans le processus politique. Ils permettent à la classe marchande et commerciale de la ville de s'exprimer auprès de l'administration municipale et ainsi de l'influencer. Compte tenu de ce rôle, on comprend peut-être mieux que le shérif ait cru que les grands jurys ne devaient être composés que de citadins. La perception du rôle des jurys par les élites réformatrices, toutefois, ne comprenait pas cette fonction représentative, d'où leur insistance pour obtenir un jury tiré de l'ensemble du district. Ce que pensaient les élites canadiennes urbaines de la diminution de leur place au sein des grands jurys à partir du début des années 1830, et surtout à partir de 1832 , reste à déterminer.

\section{CONCLUSION}

L'histoire des grands jurys au Québec et au Bas-Canada est plus complexe que celle d'une simple institution sous le contrôle des bureaucrates. Elle illustre au moins trois points fondamentaux par rapport à la citoyenneté et à la participation politique dans la colonie.

D’abord, les grands jurys sont certainement des institutions dont la représentativité est biaisée, mais pas selon la découpage bidimensionnel classique entre Britanniques / Tories et Canadiens/ Patriotes. Les Britanniques sont certainement favorisés, mais il n'y a aucune exclusion systématique des Canadiens, comme le laisserait croire le discours des réformateurs francophones et ses échos historiographiques. Quand La Minerve déclare, en 1836, que les Catholiques furent exclus des jurys entre 1763 et 1775 et "de l'administration de la justice pendant plus d'un demi-siècle», le journal a tort, tout simplement ${ }^{85}$. Au Québec et au Bas-Canada, c'est le rang social, la profession et le lieu de résidence qui déterminent avant tout la participation aux grands jurys, plus que l'appartenance ethnolinguistique ou même politique : les grands jurés représentent la bourgeoisie commerciale des villes, francophone et anglophone.

Deuxièmement, la notion que le jury ne contribue à construire qu'une identité britannique et tory, étrangère aux élites canadiennes, ne tient pas. Le jury, avec ses racines anglaises profondes, participe tout autant à l'élaboration du concept qu'à la notion de citoyenneté privilégié par les élites réformatrices. Certes, c'est un jury très différent de celui postulé par les élites tories : sa base rhétorique est la liberté et la participation populaire, 
plutôt que la constitution et le devoir des élites. C'est toutefois loin d'être un jury démocratique et républicain ou un rejet de la base britannique. N'est-ce pas là une observation qu'on pourrait faire aussi à l'endroit d'autres institutions britanniques imposées ou adoptées à la suite de la Conquête, comme le droit criminel dans son ensemble?

Enfin, les grands jurys jouent un rôle politique très important, mais pas au niveau colonial. C'est plutôt au niveau local, en l'absence d'institutions municipales représentatives, que le grand jury permet aux élites locales, canadiennes et britanniques, d'influencer une administration qui concerne directement leurs intérêts. C'est un niveau qui est le plus souvent oublié dans les analyses classiques de la vie politique de la colonie, avec comme résultat la perte d'une dimension importante de la politique qui ne suit pas les mêmes impératifs que la politique coloniale. L'importance de cette forme de représentation civique locale perdure, même après l'incorporation de Montréal en 1832, et revient en pleine force pendant la suspension de tout gouvernement représentatif à la suite des Rébellions. Ainsi, en janvier 1839, le président des Sessions trimestrielles de Montréal, François-Pierre Bruneau (cousin par alliance de Louis-Joseph Papineau et futur conseiller législatif) déclare au grand jury:

Every thing connected with the safety and health of the city, and with the public welfare in general will claim your attention and solicitude. In the present posture of our affairs, you are the only legally constituted organ and representative of the country. The country may therefore, gentlemen, justly expect from you more anxious and extended inquiry into all that affects her happiness then it was customary for your predecessors to grant, in past and better times ${ }^{86}$.

Mais même à cette époque, la fonction représentative et politique du grand jury ne fait pas l'unanimité : en 1840, Le Canadien déclare, par rapport à une représentation détaillée des Sessions trimestrielles de Québec :

Ce sont là des sujets qui méritent l'attention des autorités [...] mais qui seront probablement inutilement recommandés tant que le peuple n'influera pas directement sur la législation du pays, et c'est une recommandation que le Grand Jury a oublié de faire : avec celle-là, il aurait pu se dispenser de toutes les autres, car elle les comprend toutes ${ }^{87}$.

Dans une conception démocratique, cette institution représentative mais non élue n'avait plus sa place. 